\title{
Plastic depinning in artificial vortex channels: competition between bulk and boundary nucleation
}

\author{
T. Dröse ${ }^{1}$, R. Besseling 2 , P. Kes ${ }^{2}$, and C. Morais Smith ${ }^{3}$ \\ ${ }^{1}$ I Institut für Theoretische Physik, Universität Hamburg, D-20355 Hamburg, Germany. \\ ${ }^{2}$ Kamerlingh Onnes Laboratorium, Leiden University, P.O. Box 9504, 2300 R A Leiden, the Netherlands. \\ ${ }^{3}$ Institut de Physique Théorique, Pérolles, CH-1700 Fribourg, Switzerland.
}

(November 15, 2018)

\begin{abstract}
We study the depinning transition of a driven chain-like system in the presence of frustration and quenched disorder. The analysis is motivated by recent transport experiments on artificial vortexflow channels in superconducting thin films. We start with a London description of the vortices and then map the problem onto a generalized Frenkel-Kontorova model and its continuous equivalent, the sine-Gordon model. In the absence of disorder, frustration reduces the depinning threshold in the commensurate phase, which nearly vanishes in the incommensurate regime. Depinning of the driven frustrated chain occurs via unstable configurations that are localized at the boundaries of the sample and evolve into topological defects which move freely through the entire sample. In the presence of disorder, topological defects can also be generated in the bulk. Further, disorder leads to pinning of topological defects. We find that weak disorder effectively reduces the depinning threshold in the commensurate phase, but increases the threshold in the incommensurate phase.
\end{abstract}

PACS numbers: 74.60.Ge, 71.45.Lr, 83.50.Lh

\section{INTRODUCTION}

The depinning transition in driven systems has attracted a great deal of attention recently. The phenomenon can be observed in charge density wave systems submitted to an electric field, 1 in magnetic bubbles moving under an applied magnetic field ostadient, in current driven Josephson junction arrays, the two-dimensional electron gas in a magnetic field, which at low densities forms o Wigner crystal, but can move under an applied voltage. 1 Depinning is also important to understand tribology and solid friction, 6 surface growth of crystals with quenched bulk or substrate disorder, and the dynamics of domain walls in incommensurate solids. 7 A very prominent example of driven systems displaying a depinning transition are vortices in type-II superconductors.8

The $H-T$ phase diagram of type-II superconduf tors displays regions of both elastic and plastic flow 0 . The interplay between the elasticity of the vortex lattice and the impurities present in the substrate leads to a rich phenomenology with many static and dynamic phases. A crucial question in both the dynamics and statics is whether - in addition to thermal fluctuations - quenched disorder produces topological defects in the periodic structure. Whereas in the absence of topological defects it is sufficient to consider only elastic deformations with depinning causing elastic flow, the dynamics will be governed by plastic flow, if topological defects exist. One expects plastic motion to become important for either strong disorder, high temperature, or near the depinning transition in low dimensions. In these cases, depinning is observed to occur through "plastic channels" between pinned regions. This type of plastic flow has been found in numerical simulations of a twodimensional thin film geometry.10 12 Above the thresh- old, the filamentary channels become both denser and broader when the driving force is increased. Measurements of the differential resistance of MoGe films display abrupt steps, which could be interpreted in terms of plastic depinning. 13 Other experimental observations that here been attributed to plastifflow are the peak effect, 14,15 unsual broadband noise, 16 and steps in the $I-V$ curve. 17

In order to study the plastic depinning of vortices artificial easy-flow channels have been manufactured.18. 19 The samples are typically made of type-II superconducting thin films, which consist of a fairly strong pinning layer on top of a weakly pinning base layer. The artificial vortex flow channels are fabricated by etching away stripes of the stronger pinning top layer. In the Shubnikov phase, vortices penetrate the sample. Applying a current perpendicular to the channel direction, the resulting Lorentz force in the direction of the channel drives the vortices. Since the influence of point-like material defects in the weak pinning channel is negligible, channel vortices are mainly pinned indirectly via the interaction with the stronger pinned vortices in the channel environment. Above a threshold force, plastic depinning of the vortices inside the channel takes place. In contrast to natural channels, the depinning threshold force displays interesting oscillations when the externally applied magnetic field is varied. The position of the threshold force maxima hint at commensurability effects between the vortex lattice in the environment and in the channel.20 The magnitude of the depinning force minima and maxima indicate that lattice distortions produced by quenched disorder in the pinned channel environment is relevant in these samples.2]

In this work, we present theoretical studies of the depinning and dynamical behavior of vortices in artificial easy-flow channels. Frustration and disorder are essen- 
tial ingredients for determining the depinning transition in this driven system. Motivated by recent experiments on vortex-flow channels, we develop a model which takes into account inhomogeneities, such as the sample boundaries and disorder. We find that depinning occurs due to boundary or bulk nucleation, respectively, in the absence or presence of disorder. Further, we clarify the role of disorder and frustration and compare our results to the available experimental data.

First, we study this problem considering a perfectly ordered vortex-lattice in the environment. Starting from a London description of vortices inside and outside the channel, we derive the coefficients of a generalized Frenkel-Kontorova model.22 The force-velocity curve of this simple model displays a drastic decrease of the threshold pinning force when topological defects enter the system via the system boundaries. However, the experimental data show a smooth variation of the pinning force as a function of the magnetic field. The behavior suggests that disorder in the channel edges may lead to smoothening of the transition from the topologically ordered to the defective state. In order to investigate the transition in presence of disorder, we take into account that the vortex lattice in the channel environment is distorted due to impurities in the superconducting substrate. To determine the characteristic current-voltage or force-velocity curves, the problem is most conveniently tackled with numerical molecular dynamics simulations. This method enables us also to nicely visualize how depinning occurs in the topologically ordered and defective regimes. It turns out that systems that are topologically ordered in the static phase depin at the boundary or by generating mobile defect droplets at weak spots in the random channel potential. In the defective static phase, the pre-existing topological defects are pinned by the random potential. Then, the depinning transition occurs via a release of these defects at the threshold.

The paper is organized as follows: In Section II, we discuss the experiments that motivated the theoretical studies; in Section III we introduce the model and present the results concerning the depinning transition. Finally, the discussion and conclusions are presented in Section IV.

\section{EXPERIMENTAL MOTIVATION}

Let us start with a short overview of the experimental status of art. In order to obtain a more detailed understanding of the depinning process in disordered superconducting films experimentalists fabricated artificial vortex channels.18. 19 A sketch of a typical device is presented in Fig. 1. The devices are manufactured by first absorbing a thin layer of $\mathrm{NbN}$ (strong pinning material) on top of a $\mathrm{Nb}_{3} \mathrm{Ge}$ film (weak pinning) and then etching away a few hundred channels of width $w$ from the top layer. In the strongly pinned region, the vortex-lattice spac-

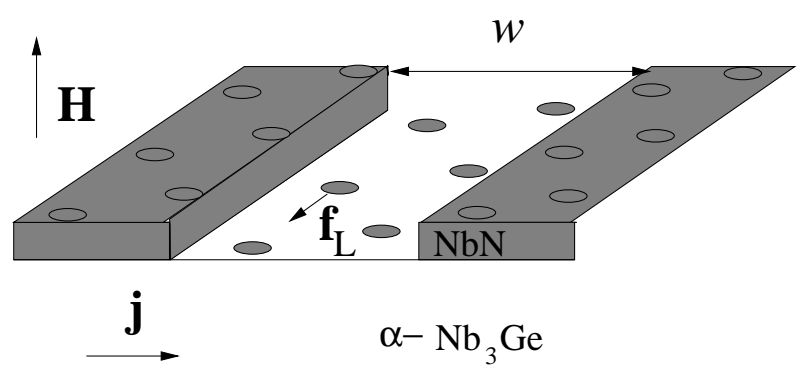

FIG. 1. Schematic plot of the artificial flow channel geometry. The channels are manufactured by etching away the strongly pinning top layer material (grey area) in stripes of width $w$. Applying a magnetic field $\mathbf{H}$ generates vortices. In the strong pinning area the vortices are static. Vortices in the weakly pinning channel are mainly pinned due to the interaction with the strongly pinned ones in the channel edges (grey area). In presence of a current density $\mathbf{j}$ a Lorentz force $\mathbf{f}_{L}$ acts on the vortices. At the depinning transition the channel vortices start to flow whereas the strongly pinned vortices in the channel environment remain static.

ing is $a_{0}=(4 / 3)^{1 / 4}\left(\Phi_{0} / B\right)^{1 / 2}$, such that $a_{0} b_{0}=\Phi_{0} / B$, with $\Phi_{0}$ the flux quantum and $b_{0}=(\sqrt{3} / 2) a_{0}$, see Fig. 2 a. In order to measure the effect of a single vortex row in the channel, the channel width $w$ is chosen to be of the order of $a_{0}$. Therefore, the vortex-lattice spacing in the channel is $a=\Phi_{0} / w B$. Depending on the mismatch parameter $\chi=\left(a / a_{0}\right)-1=\left(b_{0} / w\right)-1$, a commensurateincommensurate transition can be observed. In the experiments described here, $a_{0} \sim w \sim 100 \mathrm{~nm}$ and the distance between the channels is $10 \mu \mathrm{m}$. Due to the material steps at the channel edges, screening currents flow along the surface which interact with the vortices in the channels. As a consequence, we can expect the first rows of strongly pinned vortices near the channel edges to be lined up at fairly straight along the channel. Therefore, we can assume that the transversal shifts are negligible. On the other hand, longitudinal displacements may occur due to the presence of impurities in the superconducting material [see Fig. 2(b)]. Here we will concentrate on the longitudinal dynamics of the vortices within the channel.

In the channel, pinning due to impurities is very weak. Below the depinning threshold, vortices in the channel are localized due to the interaction with the vortices in the static environment. In the presence of a current density $\mathbf{j}$, a Lorentz force $\mathbf{f}_{L}$ acts on the vortices. As long as the vortices are pinned, the supercurrent is flowing dissipation-less through the sample. At a threshold force $f_{c}$ the channel vortices depin and start to move. The vortex motion causes dissipation of energy and a voltage drop occurs across the sample boundaries. Hence, a welldefined critical current density $j_{c}$ can be determined by measuring the current-voltage characteristics of the sample. Fig. 3 shows shear force density data deduced from current-voltage measurements.23 The oscillations in the shear force density $F_{s}$ as a function of $B$ are related to 
(a)

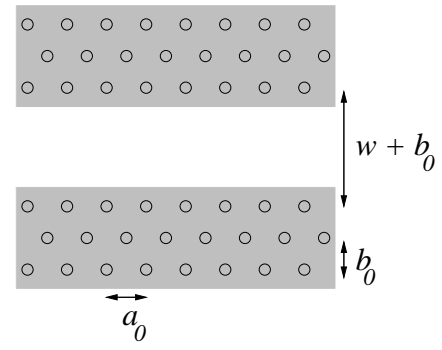

(b)

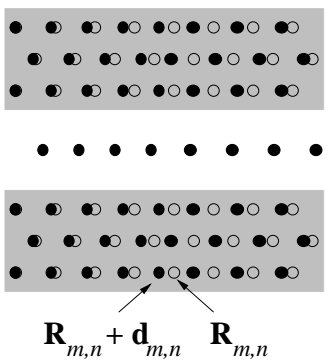

FIG. 2. (a) Easy flow channel environment (shaded) in the absence of disorder. Vortex positions in the environment are marked by open circles. (b) Immobile vortices (dots) in the environment in the presence of weak disorder and one row of mobile vortices in the flow channel.

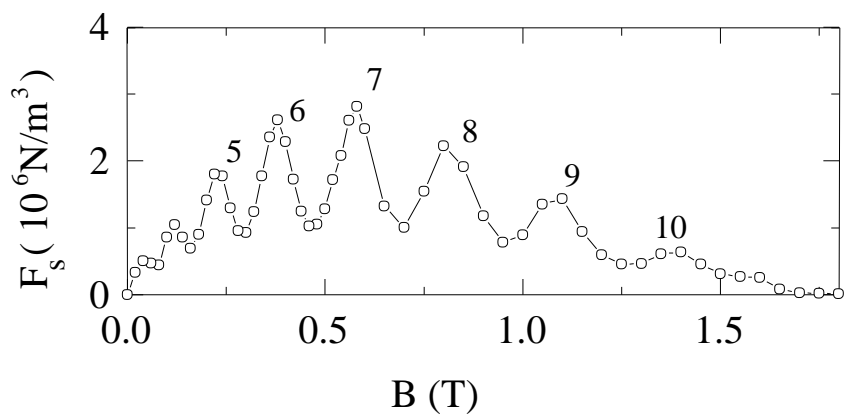

FIG. 3. The experimentally obtained critical shear force density $F_{s}$ versus magnetic field $B$ for a hannel sample with $w \approx 290 \mathrm{~nm}$ at a temperature $T=1.7 \mathrm{~K} 23$.

the (in) commensurability of the vortex lattice $b_{0}$ with the channel width $w$. Naively, we would expect the maxima of $F_{s}$ to occur at integer values of the ratio $w / b_{0}$, where the vortex lattice spacing inside the channel is commensurate with the width of the channel. $F_{s}$ should then be reduced for non-integer $w / b_{0}$. This could qualitatively be explained by the derepopment of misfit dislocations along the channel edges 18.19 Although this intuitive picture holds for the case of weak (or zero) disorder, these features change drastically in the limit of a strongly disordered environment. Indeed, as it will be explained in Ref. [23], in the latter limit, the minima and not the maxima of $F_{s}$ correspond to an integer number of vortex-rows in the channel. In this paper, however, we restrict ourselves to the limit of weakly disorderd environment and investigate the competition between boundary and bulk nucleation for activating the depinning process.

\section{A MODEL FOR ARTIFICIAL VORTEX CHANNELS}

Starting from a London description of vortices in a type-II superconductor, we now derive a simplified 1d

model for vortices in an artificial easy-flow channel.

\section{A. Channel with a perfect vortex lattice in the environment}

Let us first consider a row of straight vortices that are aligned along the $x$-axis at positions $m a_{0} \mathbf{e}_{x}$, where $m$ is an integer, $a_{0}$ the distance between adjacent vortices and $\mathbf{e}_{x}$ the unit vector in the $x$-direction. In a typeII superconductor within London theory the interaction energy between two straight vortices of length $l$ at $\mathbf{r}$ and $\mathbf{0}$ is $U(\mathbf{r})=U_{0} K_{0}(r / \lambda)$, where $U_{0}=\Phi_{0}^{2} l /\left(8 \pi^{2} \lambda^{2}\right)$. Here $\lambda$ is the penetration depth, $\Phi_{0}=h c / 2 e$ is the flux quantum, and $l \gg \lambda$ the sample thickness. Then the potential energy $E_{n}^{o}(\mathbf{r})$ that is felt by a test vortex at position $\mathbf{r}=(x, y)$ interacting with a row of vortices placed at $n b_{0} \mathbf{e}_{y}$ is

$$
E_{n}^{o}(\mathbf{r})=\sum_{m} U\left(\mathbf{r}-\mathbf{R}_{m, n}\right)
$$

where we introduced the lattice vectors $\mathbf{R}_{m, n}=([m+$ $n / 2] a_{0}, n b_{0}$ ) of a hexagonal lattice. Fourier transforming and using the Poisson sum rule the latter can be recast into

$$
E_{n}^{o}(\mathbf{r})=\sum_{\nu} \frac{\cos \left[q_{\nu}\left(x-n a_{0} / 2\right)\right]}{2 \pi a_{0}} \int_{-\infty}^{+\infty} d k_{y} \tilde{U} e^{i k_{y}\left(y-n b_{0}\right)}
$$

where $q_{\nu}=2 \pi \nu / a_{0}$ with integer $\nu$ and $\tilde{U}$ is the Fourier transform of the potential, which is given by

$$
\tilde{U}\left(q_{\nu}, k_{y}\right)=\frac{2 \pi U_{0}}{q_{\nu}^{2}+k_{y}^{2}+\lambda^{-2}} .
$$

Performing the integral over $k_{y}$ leads to

$$
E_{n}^{o}(\mathbf{r})=\sum_{\nu} B_{\nu}\left(\left|y-n b_{0}\right|\right) \cos \left[q_{\nu}\left(x-n a_{0} / 2\right)\right],
$$

where

$$
B_{\nu}(y)=\frac{\pi U_{0}}{a_{0} q_{\nu}^{\prime}} \mathrm{e}^{-q_{\nu}^{\prime} y}
$$

and $q_{\nu}^{\prime}=\sqrt{q_{\nu}^{2}+\lambda^{-2}}$.

We construct an easy flow channel by building a two dimensional vortex lattice, but leaving a region of width $w$ along the $x$-axis empty (see Fig. 2a).

If we consider a hexagonal vortex lattice in the channel environment, the potential in the channel is

$$
E_{o c}(\mathbf{r})=\sum_{n=1}^{\infty}\left[E_{n}^{o}(\mathbf{r}-\mathbf{b} / 2)+E_{-n}^{o}(\mathbf{r}+\mathbf{b} / 2)\right],
$$

where $\mathbf{b}=\left(w-b_{0}\right) \mathbf{e}_{y}$. Summing over the vortex rows $n$ one finds 


$$
E_{o c}(\mathbf{r})=\sum_{\nu} A_{\nu}(y) \cos \left(q_{\nu} x\right),
$$

the Fourier coefficients $A_{\nu}(y)$ read

$$
A_{\nu}(y)=\frac{2 \cosh \left(q_{\nu}^{\prime} y\right) B_{\nu}\left(\frac{w-b_{0}}{2}\right)}{(-1)^{\nu} \mathrm{e}^{q_{\nu}^{\prime} b_{0}}-1} .
$$

Since the magnetic inductions $B$ used in the experiments cover the entire range up to the upper critical one $B_{c 2}$, a more general expression for $A_{\nu}$ than the London limit that has been discussed until now is needed. However, the theory presented here can easily be extended to larger magnetic inductions. First one takes into account the finite diameter of the vortex core which is of the order $\xi$ in the vortex-vortex interaction potential, $U \rightarrow U_{0}\left[K_{0}(r / \lambda)-K_{0}(r / \xi)\right]$. Second, one replaces $\lambda \rightarrow$ $\lambda^{\prime}=\lambda /\left(1-B / B_{c 2}\right)^{1 / 2}$ and $\xi \rightarrow \xi^{\prime}=\xi /\left(2-2 B / B_{c 2}\right)^{1 / 2}$ to take into account the reduction of the superconducting order parameter at large magnetic fields.24 We then obtain

$$
\begin{aligned}
A_{\nu}(y) & =\frac{\pi U_{0}\left(1-B / B_{c 2}\right)}{a_{0}}\left[\frac{2 \cosh \left(q_{\nu}^{\prime} y\right) \mathrm{e}^{-q_{\nu}^{\prime} y\left(w-b_{0}\right) / 2}}{q_{\nu}^{\prime}\left[(-1)^{\nu} \mathrm{e}^{q_{\nu}^{\prime} b_{0}}-1\right]}\right. \\
& \left.-\frac{2 \cosh \left(q_{\nu}^{\prime \prime} y\right) \mathrm{e}^{-q_{\nu}^{\prime \prime} y\left(w-b_{0}\right) / 2}}{q_{\nu}^{\prime \prime}\left[(-1)^{\nu} \mathrm{e}^{q_{\nu}^{\prime \prime} b_{0}}-1\right]}\right]
\end{aligned}
$$

where now $q_{\nu}^{\prime}=\sqrt{q_{\nu}^{2}+\left(\lambda^{\prime}\right)^{-2}}$ and $q_{\nu}^{\prime \prime}=\sqrt{q_{\nu}^{2}+\left(\xi^{\prime}\right)^{-2}}$.

\section{B. Equation of motion}

The overdamped dynamics of a vortex with index $m$ at position $\mathbf{r}_{m}$ inside the channel is described by

$$
\eta \dot{\mathbf{r}}_{m}=f \cdot \mathbf{e}_{x}-\nabla E_{o c}\left(\mathbf{r}_{m}\right)-\sum_{n \neq m} \nabla U\left(\mathbf{r}_{m}-\mathbf{r}_{n}\right),
$$

where $f=j \Phi_{0} / c$ is the magnitude of the Lorentz force that drives the vortices in presence of a current density $j$. The viscous drag coefficient $\eta$ is related to the flux flow resistivity $\rho_{f f}$ by $\eta=B \Phi_{0} /\left[c^{2} \rho_{f f}(B)\right]$. The sum is taken over the positions $n$ of all other vortices inside the channel.

In the simplest case the channel width is $w \approx b_{0}$ such that only a single vortex row is inside the channel. Then the motion of the mobile vortices in the $y$-direction is essentially guided by the channel potential, whereas the interaction between vortices in the channel does not contribute significantly to the motion in the $y$-direction, $\eta \dot{y}_{m} \approx-\partial_{y} E_{o c}(\mathbf{r})$. However, the motion in the $\mathrm{x}$-direction is determined by both the interactions between mobile vortices and the gradient of the channel potential. To simplify matters, we neglect the motion in the $y$-direction, $y_{m}=0$, such that the equation of motion can be simplified to a one-dimensional one. Further, we restrict our considerations to $\lambda \gtrsim a_{0}$ as in a typical experiment. Then, the amplitudes $A_{\nu}(0)$ fall off exponentially fast, $A_{\nu}(0) \sim U_{0} \exp (-\pi \sqrt{3} \nu)$, and the approximation to consider only the first harmonic $q=q_{1}=2 \pi / a_{0}$ of the channel potential is a good one. Introducing $\mu=2 q\left|A_{1}(0)\right|$, and restricting the interaction between vortices in the channel to next-neighbor springlike forces, the equation for the overdamped longitudinal motion reads

$$
\eta \dot{x}_{m}=-\frac{\partial V}{\partial x_{m}}
$$

where the potential energy of the vortices in the channel is given by

$$
\begin{aligned}
V & =\sum_{m}\left\{\frac{\mu}{q}\left[1-\cos \left(q x_{m}\right)\right]-f x_{m}\right. \\
& \left.+\sum_{n} \frac{\kappa_{n}}{2}\left(x_{m+n}-x_{m}-n a\right)^{2}\right\} .
\end{aligned}
$$

It has the form of a generalized Frenkel-Kontorova model.22 The interactions between vortices inside the channel are approximated by Hookian springs with spring constants $\kappa_{n}=\left(U_{0} / \lambda^{2}\right) K_{0}^{\prime \prime}(n a / \lambda)$, where the double prime denotes the second derivative. Notice that the displacements $x$ are measured with respect to the vortexlattice preferred position $a$ in the channel.

The Frenkel-Kontorova model has been intensively studied close to equilibrium, $f \sim 0$, see Ref. [25]. The reduced dimensionless elasticity

$$
g=\frac{1}{q \mu} \sum_{n=1}^{\infty} n^{2} \kappa_{n} \approx \frac{e^{\pi \sqrt{3}}}{8 \pi} \frac{\lambda}{a} \gg 1
$$

together with the winding number $\Omega=a / a_{0}$ crucially determine the behavior of the system. For rational $\Omega$ the vortex chain is commensurate with the periodic channel potential whereas for irrational $\Omega$ it is in an incommensurate state. The commensurate-incommensurate transition is a continuous transition that occurs at finite mismatches $\chi$ (recall that $\left.\chi=\left(a-a_{0}\right) / a_{0}\right)$, since the creation of discommensurations costs energy.

If the discreteness of the chain is relevant, PeierlsNabarro barriers may exist. The Peierls-Nabarro is the energy barrier that has to be overcome for a translation, $x_{m} \rightarrow x_{m+1}$. Whereas this barrier is always finite for rational $\Omega$, it may vanish in the incommensurate state: if $g$ is lower than a critical value, $g_{c}\left(a / a_{0}\right)$, the incommensurate state is pinned, however, for $g>g_{c}\left(a / a_{0}\right)$, the Aubry transition to a sliding state with truly vanishing critical force takes place.26 For pinned defective configurations the Peierls-Nabarro barrier, which determines the corresponding pinning force $f_{P N}$, depends on $g$. Large $g \gg 1$ imply that an isolated defect having a size $\sim l_{d}$ extends over several lattice constants. Then, the Peierls-Nabarro barrier is nearly vanishing 25 and the pinning force is

$$
f_{P N} \approx 64 \pi^{2} g \mu \exp \left(-\pi^{2} \sqrt{g}\right) .
$$


Since $f_{P N} \ll 10^{-4} \mu$ we neglect it in the following.

Since we are most interested in the regime $g \gg 1$, it is convenient to study the model in the continuum limit. Further, we take into account the finite length of the system and consider open boundary conditions. Rewriting Eq. (12) in terms of the displacements of the vortices from the environmental lattice positions, $u_{m}=x_{m}-m a_{0}$ and substituting $m a_{0} \rightarrow x, u_{m} \rightarrow u(x)$, $\left(u_{m+n}-u_{m}\right) /\left(n a_{0}\right) \rightarrow \partial_{x} u(x), \sum_{m} \rightarrow \int d x / a_{0}$, we obtain the continuum equation of motion

$$
\begin{aligned}
\eta \dot{u}(x) & =-a_{0} \frac{\delta V[u]}{\delta u(x)}=\tilde{\kappa} \frac{\partial^{2} u(x)}{\partial x^{2}}-\mu \sin [q u(x)] \\
& +f+\chi \tilde{\kappa}[\delta(x-L)-\delta(x)]
\end{aligned}
$$

where we have introduced $\tilde{\kappa}=2 \pi a_{0} \mu g$ and the last term is an effective surface force that arises at the open sample boundaries in the presence of frustration ( $L$ is the channel length). The energy functional reads

$V[u]=\int \frac{d x}{a_{0}}\left\{\frac{\tilde{\kappa}}{2}\left(\frac{\partial u}{\partial x}-\chi\right)^{2}+\frac{\mu}{q}[1-\cos (q u)]-f u\right\}$,

where the integral runs from 0 to $L$. It can be decomposed into

$$
V=V_{S G}+V_{\chi}+V_{0}
$$

where

$$
V_{S G}[u]=\int \frac{d x}{a_{0}}\left\{\frac{\tilde{\kappa}}{2}\left(\frac{\partial u}{\partial x}\right)^{2}+\frac{\mu}{q}[1-\cos (q u)]-f u\right\}
$$

is the energy functional of the sine-Gordon model,

$$
V_{\chi}[u]=-\frac{\chi \tilde{\kappa}}{a_{0}}[u(L)-u(0)]
$$

is the frustration energy due to the mismatch $\chi$ determined by the values of the displacement field $u(0)$ and $u(L)$ at the boundaries, and $V_{0}$ is an irrelevant offset that is omitted in the following.

\section{Commensurate-Incommensurate Transition}

We now present a short review of the commensurateincommensurate transition. Then, we will extend the picture to discuss the role of edge barriers for defects in finite systems, which is crucial to understand how discommensurations penetrate a sample in the absence of thermal or quantum fluctuations.

The extrema of $V[u]$ are found solving the variational problem $\delta V / \delta u=0$. In the absence of frustration, $\chi=0$, and for $f=0$, uniform static solutions exist. They are the stable, $u_{s, n}=a_{0} n$, unstable, $u_{u, n}=(2 n+1) a_{0} / 2$, and kinked

$$
u_{k, n}\left(x ; x_{c}\right)=u_{s, n}+\frac{4}{q} \arctan \left[\exp \left(\frac{x-x_{c}}{l_{d}}\right)\right],
$$

solutions of the sine-Gordon model at zero driving force. Here, $l_{d}=a_{0} \sqrt{g}$ is the width of the kink. The corresponding anti-kink solution reads $u_{a, n}\left(x ; x_{c}\right)=$ $u_{k, n}\left(-x ;-x_{c}\right)$. The commensurate-incommensurate transition is a transition that occurs in equilibrium $(f=$ 0 ) when it becomes energetically favorable to have a finite density of discommensurations in the system. Neglecting effects of system boundaries, the mismatch $\chi_{c i}$ at which the commensurate-incommensurate transition takes place is then quickly found by comparing the energy of the kinked solution with the energy of the stable homogeneous one $V\left[u_{k, n}\right]-V\left[u_{s, n}\right]=(4 / \pi)(\tilde{\kappa} \mu / q)^{1 / 2}-\tilde{\kappa} \chi=0$,

$$
\chi_{c i}=\frac{4}{\pi}\left(\frac{\mu}{q \tilde{\kappa}}\right)^{1 / 2}=\frac{2}{\pi^{2} \sqrt{g}} .
$$

Though at the commensurate-incommensurate transition $u_{k, n}$ or $u_{a, n}$ have the same energy as $u_{s, n}$, a barrier has to be overcome in order to make the transition from $u_{s, n} \rightarrow u_{k, n}$ or $u_{s, n} \rightarrow u_{a, n}$, see upper curve in Fig. 4. In the absence of fluctuations, as considered here, discommensurations can only enter the system when this barrier vanishes (lower curve in Fig. 4). In general, the barrier vanishes at a sufficiently strong frustration or driving force. We therefore define a threshold frustration $\chi_{c}(f)$, which is a function of the driving force $f$. Finite driving forces $f>0$ are considered in Section [IID, where depinning is studied. In the following we investigate how (anti-)kinks enter the system at equilibrium $f=0$ and determine the zero-force threshold $\chi_{c}(0)$.

Let us first determine the energy that is needed to deform a uniform state into a kinked one. We note that due to frustration kinks can only be created spontaneously at the system boundaries, $x=0$ or $x=L$. Of course, in the presence of thermal fluctuations, quantum fluctuations, or quenched disorder, deep in the bulk kinks can in principle be created in the form kink-anti-kink pairs. However, in the absence of fluctuations as considered here this is not possible. The reason is that kink-anti-kink pairs cannot gain frustration energy for a spontaneous kink emergence, since $u(0)=u(L)=u_{s, n}$ and hence $V_{\chi}=0$. Thus, (anti-)kinks can only enter the system at $x=0$ and $x=L$. In the following we discuss the penetration of an anti-kink at $x=0$, having in mind that the same holds for $x=L$ and for kinks. Note that in a finite system with boundaries at $x=0$ and $x=L$ one has $\lim _{x_{c} \rightarrow-\infty} u_{a, n}\left(0 ; x_{c}\right)=u_{s, n}$. Hence, a deformation from $u_{s, n}$ to $u_{a, n}$ can be achieved by pushing an anti-kink centered at $x_{c}$ from $x_{c}=-\infty$ to $x_{c}>0$. The energy of the anti-kink solution relative to the uniform one as a function of center coordinate $x_{c}$ is then

$$
\begin{aligned}
\Delta V\left(x_{c}\right) & =V\left[u_{a, n}\left(x ; x_{c}\right)\right]-V\left[u_{s, n}(x)\right] \\
& =a_{0}^{-1} \tilde{\kappa}\left\{\chi\left[u_{a, n}\left(0 ; x_{c}\right)-u_{a, n}\left(L ; x_{c}\right)\right]\right.
\end{aligned}
$$




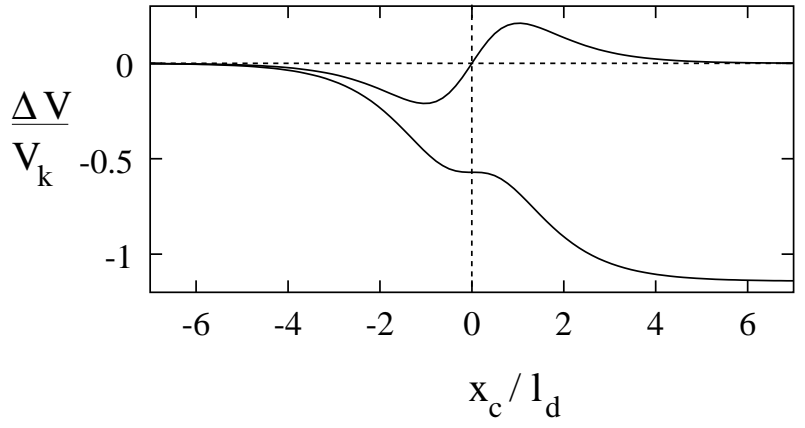

FIG. 4. Potential energy of an (anti-)kink $\Delta V$ in units of $V_{k}=\tilde{\kappa} \chi_{c i} / 2$ as a function of the (anti-)kink center $x_{c}$. Displayed are values close to the system boundary at $x=0$ for frustration parameters $|\chi|=\chi_{c i}$ (upper curve) and $|\chi|=\chi_{c}(0)$ (lower curve). At the classical commensurate-incommensurate transition, where $|\chi|=\chi_{c i}$, an entry barrier has to be overcome with the help of fluctuations to make a transition from $u_{s, n}$ to $u_{k, n}$ or $u_{a, n}$. The entry barrier vanishes at $|\chi|=\chi_{c}(0)$ where (anti-)kinks can penetrate the system.

$$
\left.+\int_{0}^{L} d x\left[\frac{\partial u_{a, n}\left(x ; x_{c}\right)}{\partial x}\right]^{2}\right\}
$$

Since the tail of the kink falls off exponentially, we can neglect the influence of the boundary at $x=L \gg l_{d}$. For simplicity, we then consider a semi-infinite system, $L \rightarrow \infty$, and obtain

$$
\begin{aligned}
\Delta V\left(x_{c}\right) & =\frac{\tilde{\kappa} \chi_{c i}}{2}\left\{\frac{4}{\pi} \frac{\chi}{\chi_{c i}} \arctan \left[\exp \left(\frac{x_{c}}{l_{d}}\right)\right]+1\right. \\
& \left.+\tanh \left(\frac{x_{c}}{l_{d}}\right)\right\} .
\end{aligned}
$$

Minimizing $\Delta V$ with respect to $x_{c}$ we find a minimum at

$$
x_{c, 1}=l_{d} \ln \left(-\frac{\pi \chi_{c i}}{2 \chi}-\sqrt{\frac{\pi^{2} \chi_{c i}^{2}}{4 \chi^{2}}-1}\right)
$$

and a maximum at

$$
x_{c, 2}=l_{d} \ln \left(-\frac{\pi \chi_{c i}}{2 \chi}+\sqrt{\frac{\pi^{2} \chi_{c i}^{2}}{4 \chi^{2}}-1}\right) .
$$

At the frustration

$$
\chi=-\chi_{c}(0)=-\frac{\pi}{2} \chi_{c i}=-\frac{1}{\pi \sqrt{g}},
$$

the minimum and the maximum merge into a saddle point at $x_{c}=0$ where the entry barrier vanishes and an anti-kink flows freely into the system. It is interesting to note that for $\chi<0$ the minimum of the anti-kink energy relative to the uniform solution is always negative, $\Delta V\left(x_{c, 1}\right)<0$. This means that in a frustrated system the uniform solution $u_{s, n}$ is unstable in the presence of a boundary. Instead, the stable solution is a virtual anti-kink with a center $x_{c}$ localized outside the system at $x_{c, 1}<0$. At the boundary, the chain thus tries to adapt optimally to the frustration to reduce its energy. For $\chi=-\chi_{c}(0)$ one finds $x_{c}=0$, which means that half of the kink is already inside the system and that it can gain more energy by sliding towards the center of the system. The scenario is the same for an anti-kink entering the system at $x=L$. For a kink the description given above is identical except that $\chi>0$. The kink entry barrier vanishes at $\chi=\chi_{c}(0)$.

The picture of the commensurate-incommensurate transition is thus drastically modified in the presence of system boundaries when there are no physical mechanisms like thermal or quantum fluctuations that are needed to cross the edge-barrier. In fact, since in the absence of fluctuations a system with boundaries remains commensurate for $|\chi|<\chi_{c}(0)$, we identify the threshold at $|\chi|=\chi_{c}(0)$ with the commensurate-incommensurate transition of a finite, purely mechanical system.

\section{Depinning in the presence of boundaries}

In the following we investigate how the chain inside the channel actually depins in presence of a driving force, $f>0$. In the simplest case, for $w=b_{0}$, we have a commensurate state without frustration, $a=a_{0}$ and $\chi=$ 0 . The chain locks perfectly to the potential and the threshold depinning force is

$$
f_{c}(0)=\mu
$$

If the system is frustrated, $b_{0} \neq w$, depinning occurs via mobile discommensurations which in the sine-Gordon model are represented by kinks or anti-kinks. As in the equilibrium case, for finite driving forces discommensurations enter the system when their entry barrier vanishes. Whether they are mobile or not depends on further barriers that may exist in the bulk.

In the continuous limit as discussed here, the defective state is not pinned. Hence, in the continuum model the threshold $\chi_{c}(0)$ indicates the change from a static equilibrium ground state $\left(u_{s, n}\right)$ to a mobile one $\left(u_{k, n}\right.$ or $\left.u_{a, n}\right)$.

Note that an exit barrier exists for a single kink at the other boundary (imagine the mirror image of the entry barrier as shown in Fig. 4 at the other end of the system). However, the exit barrier becomes irrelevant in the presence of further kinks. This can be easily understood by the following argument. Suppose a kink enters the system, freely flows to the other end, and then becomes trapped by the exit barrier. Then a second kink follows and interacts with the first one. If it would move "adiabatically" it would become trapped by the interaction with the first kink, which mediates the pinning force. However, the first kink would experience the interaction of the second kink, too. The resulting interaction 
force is of the same magnitude as the pinning force, but of opposite sign. Hence, the total force is zero and the first kink is released. The second becomes pinned for a while until it is released by the third and so forth. For non-adiabatically moving kinks, the successor does not even become pinned by the predecessor, it only lowers its velocity before the predecessor escapes due to the kinkkink interaction and the successor becomes pinned at the boundary.

So far we have determined the frustration strength $\chi_{c}(0)$ above which discommensurations enter the system in the absence of a driving force, $f=0$. In the presence of a driving force $f$, we can roughly distinguish between the regime above equilibrium threshold, $|\chi|>\chi_{c}(0)$, and below equilibrium threshold $|\chi|<\chi_{c}(0)$. Above equilibrium threshold, $|\chi|>\chi_{c}(0)$, depending on the sign of $\chi$, kinks or anti-kinks are present in the system, since the entry barrier for discommensurations has vanished. Neglecting the effects of the Peierls-Nabarro barriers, the threshold force has basically vanished,

$$
f_{c} \approx 0 .
$$

For $|\chi|<\chi_{c}(0)$ there are no kinks present in the system at equilibrium due to the finite entry barrier. However, at a sufficiently large driving force, the entry barrier vanishes, too. Then discommensurations enter the system at one boundary, freely flow through it and exit at the other boundary. In presence of a force the formation of kinks is similar to the kink-penetration at equilibrium we discussed in Section III d. Of course, the extremal solutions which determine the energy barrier for kink formation are different. The stable and unstable solutions of the sine-fordon model in the presence of a driving force $f$ read27

$$
u_{s, n}=a_{0} n+q^{-1} \arcsin (f / \mu)
$$

and

$$
u_{u, n}=\frac{(2 n+1) a_{0}}{2}-q^{-1} \arcsin (f / \mu),
$$

respectively.

For frustrations close to the equilibrium threshold frustration, $\chi_{c}(0)-|\chi| \ll \chi_{c}(0)$, the depinning threshold force is small, $f_{c} \ll \mu$. Let us study the depinning of an anti-kink at the left boundary $x=0$ for negative frustrations, $\chi<0$, in the presence of a small force, $0<f \ll \mu$. In the low-force limit, we can neglect the deformation of the anti-kink due to the force. The energy of the driven anti-kink relative to the uniform solution as a function of the anti-kink center $x_{c}$ is then

$$
\begin{aligned}
\Delta V\left(x_{c}\right) & \approx \frac{\tilde{\kappa} \chi}{a_{0}}\left[u_{a, n}\left(0 ; x_{c}\right)-u_{a, n}\left(L ; x_{c}\right)\right] \\
& +\frac{1}{a_{0}} \int d x\left\{\tilde{\kappa}\left[\frac{\partial u_{a, n}\left(x ; x_{c}\right)}{\partial x}\right]^{2}-f u_{a, n}\left(x ; x_{c}\right)\right\}
\end{aligned}
$$

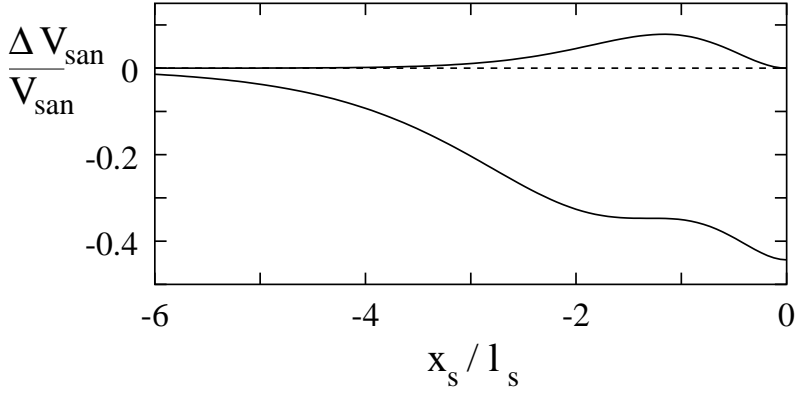

FIG. 5. Potential energy of a small amplitude nucleus $\Delta V_{\text {san }}$ in units of $V_{\text {san }}=4 \tilde{\kappa} a_{s}^{2} / 15 a_{0} l_{s}$ as a function of the position $x_{s}$ of the maximum amplitude. Shown are values close to the system boundary at $x=0$ for frustration parameters $\chi=-2 \sqrt{3} \chi_{c}(f) / 5$ (upper curve) and $\chi=\chi_{c}(f)$ (lower curve), where the barrier vanishes.

If we neglect the presence of the other boundary at $x=L$, the derivative of the potential with respect to $x_{c}$ is

$$
\begin{aligned}
\frac{d}{d x_{c}} \Delta V\left(x_{c}\right) & \approx-\frac{\tilde{\kappa}}{a_{0}} \frac{\partial u_{a, n}\left(0 ; x_{c}\right)}{\partial x_{c}}\left[\chi+\frac{\partial u_{a, n}\left(0 ; x_{c}\right)}{\partial x_{c}}\right] \\
& +\frac{f}{a_{0}} u_{a, n}\left(0 ; x_{c}\right)
\end{aligned}
$$

The anti-kink depins at the left boundary when the maximum slope of the potential at $x_{c}=0$ vanishes. This occurs at the threshold force

$$
f_{c}=\frac{4 \mu}{\pi}\left[1-\frac{|\chi|}{\chi_{c}(0)}\right]
$$

which is easy to show realizing that $u_{a, n}(0 ; 0)=a_{0} / 2$, $\partial u_{a, n}(0 ; 0) / \partial x_{c}=\chi_{c}(0)$, and $\mu=\pi \tilde{\kappa} \chi_{c}^{2}(0) /\left(2 a_{0}\right)$. The same result is found for kink depinning at the other boundary, $x=L$, for positive frustration, $\chi>0$.

At low frustration, $|\chi| \ll \chi_{c}(0)$, the depinning threshold $f_{c}$ is close to $\mu$. One thus has to consider the large force regime, $\mu-f \ll \mu$, where the lowest energy saddlepoint solution of the sine-Gordon model $u_{s a n, n}\left(x ; x_{s}\right)=$ $u_{s, n}+\Delta u\left(x ; x_{s}\right)$ has a small amplitude and hence is called small amplitude nucleus (SAN). It can be calculated by approximating the tilted cosine potential by a cubic parabola

$$
\Delta u\left(x ; x_{s}\right)=a_{s} \cosh ^{-2}\left[\frac{x-x_{s}}{2 l_{s}}\right],
$$

with center $x_{s}$, amplitude $a_{s}=3 q^{-1}[2(1-f / \mu)]^{1 / 2}$, and width $l_{s}=l_{d}[2(1-f / \mu)]^{-1 / 4}$.

Let us now consider the penetration of an anti-kink for $\chi<0$ at the boundary $x=0$. The energy difference $\Delta V_{\text {san }}=V\left[u_{\text {san }, n}\right]-V\left[u_{s, n}\right]$ as a function of $x_{s} \leq 0$ is

$$
\begin{aligned}
& \Delta V_{\text {san }}\left(x_{s}\right)=\frac{4 \tilde{\kappa} a_{s}^{2}}{15 a_{0} l_{s}}\left\{1+\frac{5}{2 \sqrt{3}} \frac{\chi}{\chi_{c}(f)} \frac{\Delta u\left(0, x_{s}\right)}{a_{s}}\right. \\
& \left.-\left[\frac{3 \Delta u\left(0, x_{s}\right)}{2 a_{s}}+1\right]\left[1-\frac{\Delta u\left(0, x_{s}\right)}{a_{s}}\right]^{3 / 2}\right\},
\end{aligned}
$$




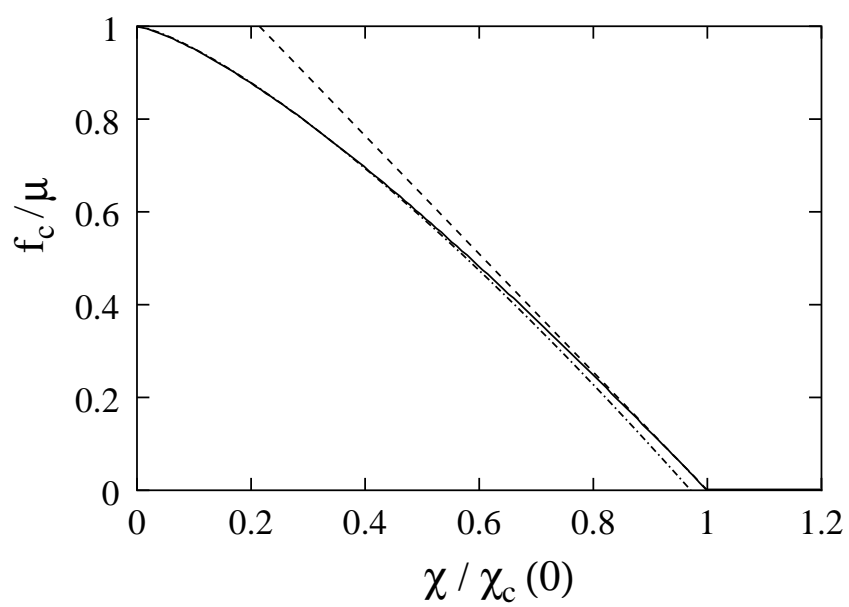

FIG. 6. Critical force $f_{c}$ as a function of the mismatch parameter $\chi$. Shown are the numerical integration results (solid line), high force approximation $f_{c}-\mu \ll \mu$ (dashed-dotted line) and the low force approximation $f_{c} \ll \mu$ (dashed line).

where

$$
\chi_{c}(f)=\frac{\pi \chi_{c i}}{2 \sqrt{3}}\left[2\left(1-\frac{f}{\mu}\right)\right]^{3 / 4} .
$$

As shown in Fig. 5, the SAN potential $\Delta V_{\text {san }}\left(x_{s}\right)$ has a barrier (upper curve), which vanishes at $\chi=-\chi_{c}(f)$ (lower curve). This can be seen by analyzing the zeros of the derivative

$$
\begin{aligned}
& \frac{d}{d x_{s}} \Delta V_{\text {san }}\left(x_{s}\right)=\frac{\tilde{\kappa} a_{s}}{a_{0} l_{s}} \frac{\partial \Delta u\left(0, x_{s}\right)}{\partial x_{s}}\left\{\frac{2}{3 \sqrt{3}} \frac{\chi}{\chi_{c}(f)}\right. \\
& \left.+\frac{\Delta u\left(0, x_{s}\right)}{a_{s}}\left[1-\frac{\Delta u\left(0, x_{s}\right)}{a_{s}}\right]^{1 / 2}\right\} .
\end{aligned}
$$

One zero is given by $x_{s}=0$, where the partial derivative $\partial \Delta u\left(0, x_{s}\right) / \partial x_{s}$ vanishes. The others can in principle be found by studying the term in the curly brackets which becomes zero if

$$
\left[\frac{\Delta u}{a_{s}}\right]^{2}\left[1-\frac{\Delta u}{a_{s}}\right]=\frac{4}{27}\left[\frac{\chi}{\chi_{c}(f)}\right]^{2} .
$$

However, to find the threshold condition, we do not need to calculate $x_{s}$ explicitely, it is sufficient to determine $\Delta u\left(0 ; x_{s}\right)$. Equation (36) has at most three roots, depending on the value of $\chi^{2}>0$. One of the roots is negative, which is no solution, since $0<\Delta u \leq a_{s}$ [see Eq. (32)]. For $-\chi_{c}(f)<\chi<0$ there are two positive roots, which indicate the existence of two extrema of $\Delta V_{\text {san }}$, a minimum and a maximum. Calculating the extrema of Eq. (36) with respect to $\Delta u$, one sees that at $\chi=-\chi_{c}(f)$ the positive roots of Eq. (36) become degenerate, which means that the minimum and the maximum merge into a saddle point of $\Delta V_{\text {san }}$. Hence, the entry barrier of the SAN vanishes. At this value, $\Delta u=2 a_{s} / 3$. Thus, two thirds of a SAN are localized at the left boundary, $x=0$, but are unstable against small perturbations. Increasing either $\chi$ or $f$ depins the SAN which then evolves into a full anti-kink. Finally, from $\Delta u\left(0 ; x_{s}\right)=2 a_{s} / 3$ we find $x_{s}=l_{s} \ln (2-\sqrt{3})$.

To summarize, for low frustrations $|\chi| \ll \chi_{c}(0)$ and for frustrations close to the equilibrium threshold $\chi_{c}(0)-$ $|\chi| \ll \chi_{c}(0)$ the threshold force $f_{c}$ is given by

$$
f_{c} \approx \begin{cases}\mu\left\{1-\frac{3^{2 / 3}}{2}\left[\frac{|\chi|}{\chi_{c}(0)}\right]^{4 / 3}\right\}, & |\chi| \ll \chi_{c}(0) \\ \frac{4}{\pi} \mu\left[1-\frac{|\chi|}{\chi_{c}(0)}\right], & \chi_{c}(0)-|\chi| \ll \chi_{c}(0) \\ 0, & |\chi| \gtrsim \chi_{c}(0) .\end{cases}
$$

For completeness, we calculated the threshold force for arbitrary frustration by numerical integration. To calculate the static and dynamic solutions of Eq. (15), we use a standard numerical integration procedure. Starting with a flat initial configuration, $u_{m}=0$, we iterate

$$
u_{m}(t+\delta t)=u_{m}(t)+\delta t v_{m}(t)
$$

with

$$
\begin{aligned}
v_{m}(t) & =f+\sin \left[2 \pi u_{m}(t)\right]+2 \pi g\left[u_{m+1}(t)+u_{m-1}(t)\right. \\
& \left.-2 u_{m}(t)+\delta_{m, M}-\delta_{m, 1}\right]
\end{aligned}
$$

where length is measured in units of $a_{0}$, time in units of $t_{0}=a_{0} \eta / \mu$, and force in units of $\mu$. The length of the system is $M=L / a_{0}$. Recalling that the vortexvortex interaction energy falls off exponentially fast for distances between vortices larger than $\lambda$, we take only the $N=\left[5 \lambda / a_{0}\right]$ next neighboring vortices into account in the sum over channel vortices. The channel has a length of $L$. At its ends, we apply boundary conditions taking into account the frustration, $\chi$. For a given force, Eq. (38) is iterated until a fairly steady state is reached, $\left[v_{m}(t+\delta t)-v_{m}(t)\right] / \delta t<10^{-4}$. Channel vortices are defined to be static, if $v_{m}<10^{-8}$. The calculated $u_{m}(t)$ and $v_{m}(t)$ are recorded for several forces. In addition we can record the particle trajectories $x_{m}(t)$ to visualize the dynamical behavior of the channel vortices close to the depinning transition. The numerical results and the analytical limits for $f_{c}(\chi)$ are shown in Fig. 6 .

The numerical integration also allows to determine the velocity averaged over time and space,

$$
v=\frac{a_{0}}{L} \sum_{m=1}^{L} \int_{0}^{T} \frac{d t}{T} \dot{u}_{m}(t),
$$

as a function of the force $f$ as shown in Fig. 7 .

Above $\chi_{c}(0)$ where the entry barrier has vanished the topological defects move freely through the sample. The linear force-velocity characteristics resembles to the one of a single free particle with dissipative dynamics, 


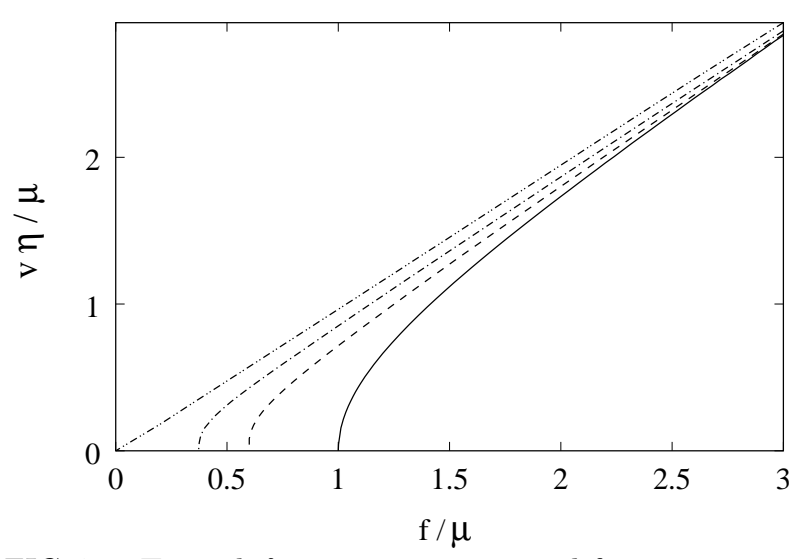

FIG. 7. Typical $f-v$ curves computed for systems with $L=1000 a_{0}$ and $\lambda=a_{0}$. The result for the unfrustrated commensurate case, $\chi=0$ (solid line), is identical to the curve of a single particle in a sinusoidal potential, $v \sim(f-\mu)^{\nu}$ with $\nu=1 / 2$. Frustration lowers the threshold force $f_{c}$, but does not alter the exponent $\nu$ in the commensurate regime. However, in the incommensurate state, $|\chi|>\chi_{c}(0)$, the $f-v$ curve becomes linear, $v \sim f$. Shown are the $f-v$ characteristics for frustrated systems with $\chi=\chi_{c}(0)$ (dash-dot-dotted), $0.7 \chi_{c}(0)$ (dash-dotted), and $0.5 \chi_{c}(0)$ (dashed).

$v=f / \eta$. The velocity of the entire chain is determined by the velocity of the defects that enter the system at the boundary. At $|\chi|=\chi_{c}$, where the entry barrier for defects has vanished, $\partial_{x}^{2} u(x=0, L)=0$. This means that close to the maximum of the sinusoidal potential, where the chain bead spends most of the time, it effectively behaves like a single particle which is driven by a force $f$. For $|\chi|>\chi_{c}$ a similar argument can be given. At $f=0$ the effective force at the system boundary pushes defects into the channel until their density is so high, that their repulsion prevents new defects to flow in. Effectively, the chain bead at the boundary reaches an unstable equilibrium. Driving the system now with a non-zero force $f>0$ results in the same motion for the bead at the boundary as for $|\chi|=\chi_{c}$.

For $\chi<\chi_{c}(0)$ the entry barrier for the defects becomes relevant and the force-velocity characteristics shows the behavior typical for quasi-particles with a vanishing saddle-point barrier with $v \sim\left[f-f_{c}(\chi)\right]^{1 / 2}$ for $f \rightarrow f_{c}$. Finally, in the absence of frustration the particles depin instantly and $v=\left(f_{c} / \eta\right)\left(2 f / f_{c}-2\right)^{1 / 2}$ like a single particle in a sinusoidal potential.

\section{E. Preliminary comparison with experimental data}

Let us now compare these theoretical findings with experimental and numerical data.20 Clearly, the generalized Frenkel-Kontorova model predicts a nearly vanishing depinning force $f_{c} \approx 0$ in the defective state. In the commensurate state, the depinning threshold $f_{c}$ is finite, but decreases monotonously with increasing frustration and vanishes at the commensurate-incommensurate transition, where the critical mismatch is reached, $\chi=\chi_{c}(0)$, see Fig. 6. As a typical value for $\lambda / a_{0}$ in the low field limit we consider $\lambda=3 a_{0}$. From Eq. (13) we find $g \sim 28$ and hence Eq. (24) yields $\chi_{c}(0) \sim 0.06$. Since $\chi=\left(b_{0} / w\right)-1$, one expects a sharp spike in the threshold force around the magnetic induction with a vortex lattice plane distance $b_{0}=w$.

If we associate the maxima of the critical force to the spikes corresponding to a commensurate state and the minima to the incommensurate one, we would nonetheless observe that some essential ingredient must be missing in our model. Indeed, in Fig. 3 we see that instead of sharp spikes, the measurements rather show a continuous modulation of the critical current as a function of the magnetic field, or equivalently, as a function of the frustration inside the channel. In addition, the data do not exhibit the vanishing pinning barriers which are expected for incommensurate (defective) structures with perfectly ordered channel edges. Both phenomena cannot be explained solely in terms of thermal or quantum fluctuations: They would lead to an effective reduction of the pinning barrier in both cases. This motivates us to study the influence of quenched disorder in the environmental vortex lattice. Actually, recent experiments have unvealed the essential role of disorder. It turns out that the picture of maximum critical force corresponding to an integer number of rows inside the channel is only valid for zero and weak disorder. If the disorder is strong, exactly the opposite will be realized, namely, the integer number of rows will correspond to the minima of $f_{c}$ (Ref. [23]).

It is generally understopd, that quenched disorder leads to pinning of vortices $\mathrm{g}$ or topological defects of the vortex lattice, which would explain the increased critical force in the defective state. It remains to understand how disorder leads to a reduction of pinning barriers in the commensurate regime.

\section{F. Channel with a distorted vortex lattice in the environment}

In a realistic sample the static nature of the channel environment is caused by some sort of pinning which may distort the vortex lattice. In the following we consider a device, where the vortices outside the channel are pinned by quenched disorder. The pinning in the channel environment is strong enough to guarantee that it remains pinned at all considered current densities whereas inside the channel pinning by quenched disorder is orders of magnitudes lower and can be entirely neglected. In a typical experiment the magnetic inductions are so large that the interaction between the vortices is much stronger than the pinning. Further, we assume that the vortex lattice in the channel environment is free of dislocations. Then it is natural to treat the vortex lattice in its elastic 
limit. In a weakly pinned vortex lattice, distortions of the order of the coherence length $\xi$ due to the disorder occur on average on a length $l_{c}$ in the direction of the magnetic field and on a length $R_{c}$ transverse to it. Here, we consider two-dimensional collective pinning where $l_{c} \gg l$ such that the approximation of straight vortex lines remains valid.

With quenched disorder in the environment of the channel, the interaction energy of a single vortex with the vortex row becomes

$$
E_{n}(\mathbf{r})=\sum_{m} U\left(\mathbf{r}-\mathbf{R}_{m, n}-\mathbf{d}_{m, n}\right)
$$

where $\mathbf{d}_{m, n}$ are the displacements from the ordered positions $\mathbf{R}_{m, n}$. It is convenient to rewrite the row potential in terms of the vortex density

$$
E_{n}(\mathbf{r})=\int d^{2} r^{\prime} U\left(\mathbf{r}-\mathbf{r}^{\prime}\right) \rho_{n}\left(\mathbf{r}^{\prime}\right)
$$

where

$$
\rho_{n}\left(\mathbf{r}^{\prime}\right)=\sum_{m} \delta\left(\mathbf{r}^{\prime}-\mathbf{R}_{m, n}-\mathbf{d}_{m, n}\right) .
$$

We introduce a continuous displacement field

$$
\mathbf{d}(\mathbf{r})=\frac{a_{0}^{2}}{4 \pi^{2}} \int_{B Z} d^{2} k e^{i \mathbf{k r}} \sum_{m, n} e^{-i \mathbf{k} \mathbf{R}_{m, n}} \mathbf{d}_{m, n}
$$

where $B Z$ indicates that the integration is restricted to the first Brillouin zone. Note that $\mathbf{d}\left(\mathbf{R}_{m, n}\right)=\mathbf{d}_{m, n}$. In order to derive a simplified one-dimensional model as in the ordered case, we make use of a few approximations. Since we consider the vortex lattice to be in the elastic limit, then

$$
|\nabla \cdot \mathbf{d}| \sim \xi / R_{c} \ll 1
$$

Moreover, since the vortex potential falls off exponentially fast for $\left|\mathbf{r}-\mathbf{r}^{\prime}\right|>\lambda$, the channel environment is mainly probed within $\left|\mathbf{r}-\mathbf{r}^{\prime}\right|<\lambda$, where one can estimate $\left|\mathbf{d}\left(\mathbf{r}^{\prime}\right)-\mathbf{d}(\mathbf{r})\right| \lesssim \lambda \xi / R_{c}$ using Eq. (45). For $\lambda \xi / R_{c} \ll a_{0} / 2$ it is then reasonable to expand the displacements $\mathbf{d}\left(\mathbf{r}^{\prime}\right)$ in the integral,

$$
\mathbf{d}\left(\mathbf{r}^{\prime}\right)=\overline{\mathbf{d}}(\mathbf{r})+\mathcal{O}\left(\frac{\lambda \xi}{R_{c}}\right)
$$

Here, we have introduced the coarse grained displacement field

$$
\overline{\mathbf{d}}(\mathbf{r})=\left(2 \pi U_{0}\right)^{-1} \lambda^{-2} \int d^{2} r^{\prime} U\left(\mathbf{r}-\mathbf{r}^{\prime}\right) \mathbf{d}\left(\mathbf{r}^{\prime}\right),
$$

which is smooth on the scale $\lambda$. Up to terms of the order $\mathcal{O}\left(\lambda \xi / R_{c}\right)$, we obtain 23

$$
E_{n}(\mathbf{r})=E_{n}^{o}[\mathbf{r}-\overline{\mathbf{d}}(\mathbf{r})]
$$

for the collectively pinned vortex row potential.

To calculate the effective channel potential for a channel of width $w \sim b_{0}$, we perform the summation over pinned vortex rows $E_{n}$ as in the ordered case. Further, since the influence of point-like disorder at the edge is assumed to be much weaker than the re-ordering due to the edge currents, we may well take $\bar{d}_{y}(\mathbf{r})=0$. Then, the interaction of a single vortex in the channel with the disordered environment reads

$$
E_{d c}(\mathbf{r})=\sum_{\nu} A_{\nu}(y) \cos \left\{q_{\nu}\left[x-\bar{d}_{x}(\mathbf{r})\right]\right\},
$$

For the partial derivatives of the channel potential one finds

$$
\begin{aligned}
& \partial_{x} E_{d c}(\mathbf{r})=-\sum_{\nu} q_{\nu} A_{\nu}(y) \sin \left\{q_{\nu}\left[x-\bar{d}_{x}(\mathbf{r})\right]\right\} \\
& \partial_{y} E_{d c}(\mathbf{r})=\sum_{\nu} A_{\nu}^{\prime}(y) \cos \left\{q_{\nu}\left[x-\bar{d}_{x}(\mathbf{r})\right]\right\}
\end{aligned}
$$

plus terms of order $\mathcal{O}\left(\lambda \xi / R_{c}\right)$. As in the perfectly ordered case, we now consider the equation of motion. Substituting $E_{o c}$ by $E_{d c}$ in Eq. (10) and following similar arguments we derive an equation for the longitudinal motion. Introducing $\tilde{\varphi}(x)=q \bar{d}_{x}(x, 0)$ we obtain a generalized phase-disordered Frenkel-Kontorova model,

$$
\begin{aligned}
V & =\sum_{m}\left\{\frac{\mu}{q}\left\{1-\cos \left[q x_{m}-\tilde{\varphi}\left(x_{m}\right)\right]\right\}-f x_{m}\right. \\
& \left.+\sum_{n} \frac{\kappa_{n}}{2}\left(x_{m+n}-x_{m}-n a\right)^{2}\right\} .
\end{aligned}
$$

The corresponding energy functional in the continuum limit is then

$$
\begin{aligned}
V[u]= & a_{0}^{-1} \int_{0}^{L} d x\left\{\frac{\tilde{\kappa}}{2}\left(\frac{\partial u}{\partial x}-\chi\right)^{2}+\frac{\mu}{q}\{1-\right. \\
& \cos [q u-\tilde{\varphi}(x+u)]\}-f u\}
\end{aligned}
$$

and the resulting equation of motion for the displacement fields $u(x, t)$ reads

$$
\begin{aligned}
\eta \dot{u} & =f-\mu \sin [q u-\tilde{\varphi}(x+u)]+\tilde{\kappa} \frac{\partial^{2} u}{\partial x^{2}} \\
& +\tilde{\kappa} \chi[\delta(x-L)-\delta(x)],
\end{aligned}
$$

where $\partial_{x} \bar{d}_{x}$-terms that are of order $\mathcal{O}\left(\lambda \xi / R_{c}\right)$ have been neglected.

\section{G. Depinning in a channel with distorted environment}

Up to now, we have not specified the disorder displacement field $\bar{d}_{x}$. To gain some basic understanding, we now consider the effect of a local distortion on the depinning 
properties. The field we choose is somewhat academic, but is convenient to understand the effect of a lattice distortion at the system boundary and in the bulk. The perturbation occurs around $x_{\varphi}$,

$$
\bar{d}_{x}(x, 0)=W\left(x-x_{\varphi}\right)\left[\theta\left(x-x_{\varphi}\right)-\frac{1}{2}\right] .
$$

Here, $\theta(x)$ is the Heaviside function and $W>0$ the distortion parameter. It is convenient to introduce transformed displacement fields $\tilde{u}=u-q^{-1} \tilde{\varphi}$. Neglecting terms of the order $\left(\partial_{x} \tilde{u}\right)^{2} \tilde{\varphi}^{\prime}$ and $\left(\tilde{\varphi}^{\prime}\right)^{2} \partial_{x} \tilde{u}$, the relevant $\tilde{u}$-dependent part of the energy functional reads

$$
\tilde{V}[\tilde{u}]=V_{S G}[\tilde{u}]+V_{\chi}[\tilde{u}]+V_{\varphi}[\tilde{u}]
$$

where

$$
V_{\varphi}[\tilde{u}]=\frac{\tilde{\kappa}}{a_{0}} \int d x \frac{\partial \tilde{u}}{\partial x} \frac{\partial \bar{d}_{x}}{\partial x} .
$$

In the following, we examine the effect of lattice distortions at the boundaries, $x_{\varphi}=0, L$, and in the bulk, $x_{\varphi}=L / 2$. To gain a basic understanding of the depinning process, we restrict the analysis to the large system limit, $L / 2 \gg l_{s}$, where the system is so large that the depinning configurations at the weak spots $x_{\varphi}=0, L$, and $x_{\varphi}=L / 2$ do not interact with each other.

For $x_{\varphi}=0$, the lattice is distorted homogeneously in the entire sample and the contribution of the distortion to the energy functional yields

$$
V_{\varphi}[\tilde{u}]=\frac{\tilde{\kappa} W}{2 a_{0}}[\tilde{u}(L)-\tilde{u}(0)] .
$$

Physically, the distortion results in an additional frustration of the system, as can be immediately understood by comparing Eq. (56) with Eq. (17). This means that for the threshold force, one can use the results that were found in the absence of the distortion [see Eq. (37)], but the frustration has to be replaced by an effective frustration

$$
\chi \rightarrow \chi-\frac{W}{2}
$$

The result is thus a simple shift of the $\chi-f_{c}$-curve. Similarly, for $x_{\varphi}=L$, the $f-\chi$-curve is shifted,

$$
\chi \rightarrow \chi+\frac{W}{2}
$$

For $x_{\varphi}=L / 2$, in addition to boundary depinning, bulk depinning at $x_{\varphi}$ can occur. In large systems, $L / 2 \gg l_{s}$ we can treat the effect of the distortions at the boundaries and in the bulk separately. At the boundaries, the threshold solution is then approximately given by the solution in absence of the defect, but with an increased effective frustration $\chi+W / 2$ in the left half and a lowered effective frustration $\chi-W / 2$ in the right half of the system. The threshold force for boundary depinning is again given by Eq. (37) with the modulus of the frustration replaced by

$$
|\chi| \rightarrow|| \chi\left|-\frac{W}{2}\right| .
$$

To understand bulk depinning, we first restrict the considerations to $\chi=0$ and then discuss the behavior in the presence of frustration.

For $L / 2 \gg l_{s}$ we can neglect the influence of the boundaries and treat them as if they were shifted to $\pm \infty$. Then, the extremal threshold solution $\tilde{u}_{\varphi}\left(x ; x_{\varphi}\right)$ can be constructed by joining two extremal solutions of $V_{S G}$. The matching condition is found from

$$
\frac{\partial u(x-\varepsilon)}{\partial x}=\frac{\partial u(x+\varepsilon)}{\partial x}
$$

such that the matching condition for the transformed field $\tilde{u}_{\varphi}\left(x ; x_{\varphi}\right)$ reads

$$
\frac{\partial \tilde{u}_{\varphi}\left(x_{\varphi}-\varepsilon ; x_{\varphi}\right)}{\partial x}-\frac{\partial \tilde{u}_{\varphi}\left(x_{\varphi}+\varepsilon ; x_{\varphi}\right)}{\partial x}=W
$$

The mirror symmetry requires $\partial_{x} u\left(x_{\varphi}\right)=0$, hence $\partial_{x} \tilde{u}\left(x_{\varphi} \pm \varepsilon\right)=\mp W / 2$. At the threshold force $f_{c}$ stable solutions cease to exist. In fact, it can be shown that at $f_{c}$ the stable solution merges with an unstable one. This occurs, when the maximum of the tongue developing at $x_{\varphi}$ reaches the maximum of the sinusoidal potential. ¿From $\delta V / \delta u=0$ follows that at the potential maximum the extremal solution has to fulfill $\partial_{x}^{2} u\left(x_{\varphi}\right)=0$, which holds if $\partial_{x}^{2} \tilde{u}\left(x_{\varphi} \pm \varepsilon\right) \rightarrow 0$ for $\varepsilon \rightarrow 0$.

For weak distortions, $|W / 2| \ll 1 /(\pi \sqrt{g})$, the threshold force for bulk depinning is close to $\mu$ and the threshold solution $\tilde{u}_{\varphi}=\tilde{u}_{s}+\Delta \tilde{u}_{\varphi}$ can be found by merging two SAN solutions at $x_{\varphi} \pm x_{a}$, where $x_{a}=l_{s} \ln (2+\sqrt{3})$,

$$
\Delta \tilde{u}_{\varphi}\left(x ; x_{\varphi}\right)=\left\{\begin{array}{cl}
\Delta u\left(x ; x_{\varphi}+x_{a}\right) & x<x_{\varphi} \\
\Delta u\left(x ; x_{\varphi}-x_{a}\right) & x \geq x_{\varphi} .
\end{array}\right.
$$

The maximum value of the tongue developing at $x_{\varphi}$ is given by $\Delta \tilde{u}_{\varphi}\left(x_{\varphi} ; x_{\varphi}\right)=2 a_{s} / 3$. This implies

$$
\frac{\partial \Delta \tilde{u}_{\varphi}\left(x_{\varphi}-\varepsilon ; x_{\varphi}\right)}{\partial x}=\frac{2 a_{s}}{3 \sqrt{3} l_{s}}=\frac{W}{2},
$$

from which one obtains the bulk depinning threshold force in the presence of weak distortions,

$$
\hat{f}_{c}=\mu\left[1-\frac{1}{2}\left(\frac{\pi \sqrt{3 g}}{2} W\right)^{4 / 3}\right]
$$

This formula becomes invalid for $2 /(\pi \sqrt{g})-|W| \ll$ $2 /(\pi \sqrt{g})$. For strong distortions, $|W| \lesssim 2 /(\pi \sqrt{g})$, the threshold configuration can be constructed by merging a kink and an anti-kink

$$
\tilde{u}_{\varphi}\left(x ; x_{\varphi}\right)= \begin{cases}u_{k}\left(x ; x_{\varphi}\right) & x<x_{\varphi} \\ u_{a}\left(x ; x_{\varphi}\right) & x \geq x_{\varphi}\end{cases}
$$


from which one obtains the bulk depinning threshold force in the presence of strong distortions,

$$
\hat{f}_{c}=\frac{4 \mu}{\pi}\left(1-\frac{\pi \sqrt{g}}{2} W\right) \text {. }
$$

At $W=2 /(\pi \sqrt{g})$ stable saddle-point solutions cease to exist for all $f$ and disorder induced mobile kink-anti-kink pairs are spontaneously formed even at equilibrium.

After having gained some understanding how pinning occurs at a weak spot in the bulk for $\chi=0$, let us now consider the frustrated case, $|\chi|>0$, where bulk depinning competes with boundary depinning. Comparing the bulk depinning threshold $\hat{f}_{c}(W)$ with the boundary threshold in presence of the defect, $f_{c}(|\chi|-W / 2)$, we find that for $|\chi|<W$ the system depins in the bulk and for $|\chi|>W$ at one of the boundaries. Note that to lowest order, we can apply these results to distortions of this kind that are not necessarily centered at $x_{\varphi}=L / 2$, as long as $l_{s} \ll x_{\varphi} \ll L-l_{s}$ holds.

\section{H. Depinning in a channel with randomly displaced edge vortices}

Opposed to the rather well behaved distortions of the previous paragraph, we now consider the effect of randomly displaced vortices in the channel edge. We mimic the disorder by uncorrelated relative displacements $\left[\bar{d}_{x}\left(\mathbf{R}_{m+1, n}\right)-\bar{d}_{x}\left(\mathbf{R}_{m, n}\right)\right] / a_{0}$. The latter are independent identically box-distributed random numbers within the interval $[-W / 2, W / 2]$,

$$
P_{W}\left(\bar{d}_{x}\right)=\frac{1}{W}\left[\theta\left(\bar{d}_{x}+W / 2\right)-\theta\left(\bar{d}_{x}-W / 2\right)\right] .
$$

The width of the box distribution $W$ parameterizes the disorder strength. Then, $\tilde{\mu}$ and $\partial_{x} \tilde{\varphi}$ are random functions, which are smooth on the scale $\lambda$ and bounded. Note that although $\partial_{x} \tilde{\varphi}$ is bounded, $\tilde{\varphi}$ is unbounded. Thus, long range order is lost along the channel direction. On length scales much larger than $a_{0}$, the displacement field $\bar{d}_{x}(\mathbf{r})$ behaves like a random walk in $1 \mathrm{D}$ and the phase-phase correlator scales linearly with the distance, $\left\langle[\tilde{\varphi}(x)-\tilde{\varphi}(0)]^{2}\right\rangle \propto x$.

The unfrustrated case has been discussed in Ref. 23. Here we generalize the results for $|\chi|>0$. In weakly frustrated systems, $|\chi|<W / 2$, nucleation still occurs at a weak spot in the bulk. Hence, the depinning threshold $\tilde{f}_{c}$ should be independent of the frustration $\chi$. This can be indeed observed, see Fig. \& the $\chi-\tilde{f}_{c}$-curve has a plateau around $\chi \sim 0$, before at larger values of $\chi$ depinning takes place via the formation of defects at the boundary as in the ordered case. The threshold for boundary depinning is affected by lattice distortions at the sample edge as discussed in Section III G resulting in an overall shift of the $\chi-\tilde{f}_{c}$-curve to lower values of $\chi$.

For intermediate frustration, $0 \ll \chi \ll \chi_{c}(0)$, a defect that entered the sample via the boundary cannot

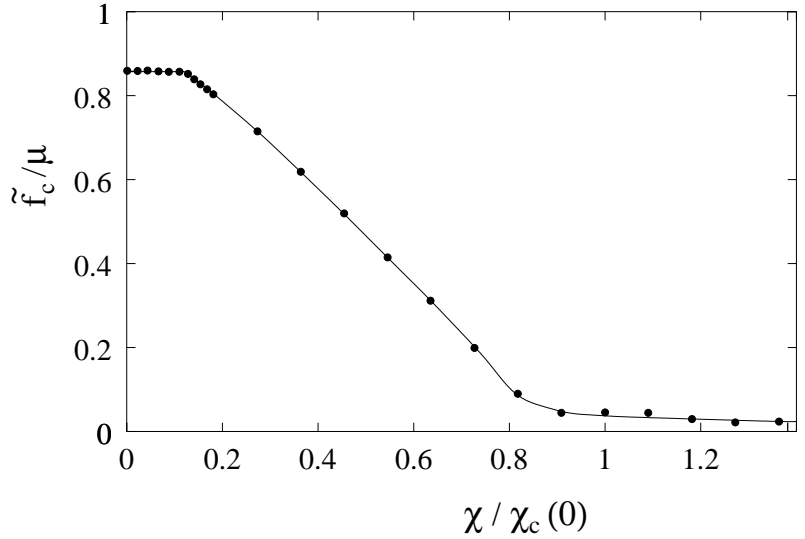

FIG. 8. Numerically obtained minimum threshold force $\tilde{f}_{c}$ as a function of frustration $\chi$ for 100 channels with $L=100 a_{0}$, $\lambda=a_{0}$, and $W=0.1$.

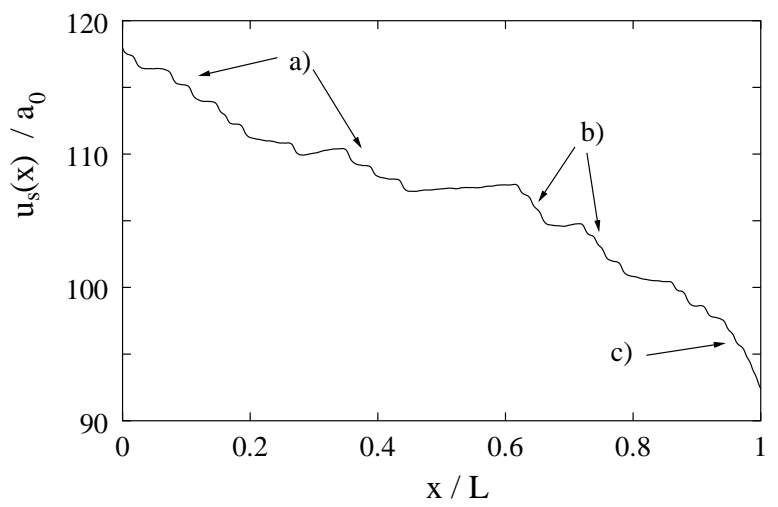

FIG. 9. Static displacements $u_{s}(x)$ in a system of length $L=1000 a_{0}$ for $\chi=-\chi_{c}(0)$ in the pinned regime, $f<\tilde{f}_{c}$. (a) Pinned single interstitials (kinks). (b) Multiple pinned kinks at strong pinning sites. (c) Kink accumulation at the end of the system, $x \lesssim L$, where in addition to disorder the exit barrier is present.

be pinned by disorder in the bulk. It moves freely to the other boundary, where it becomes pinned by the exit barrier until being released by the next defect that enters the channel and then travels freely to the exit.

For frustrations above $\chi_{c}(0)-W / 2$ bulk pinning becomes possible since the boundary depinning threshold force becomes lower than the disorder induced pinning forces in the bulk. In Fig. 8 bulk pinning becomes relevant around $\chi \sim 0.8 \chi_{c}(0)$. Indeed one can observe pinning of defects and bundles of defects in the numerical simulations in this regime. In Fig. 9 a static state in a system of length $L=1000 a_{0}$ for $\chi=-\chi_{c}(0)$ just below the depinning threshold $f \lesssim \tilde{f}_{c}$ is shown. One clearly sees single pinned kinks and a few multiple-pinned kinks (bundles). At the exit of the system kinks accumulate due to both bulk pinning and due to pinning at the presence of the exit barrier. When reaching the depinning threshold, a defect is formed at the entry on the channel 


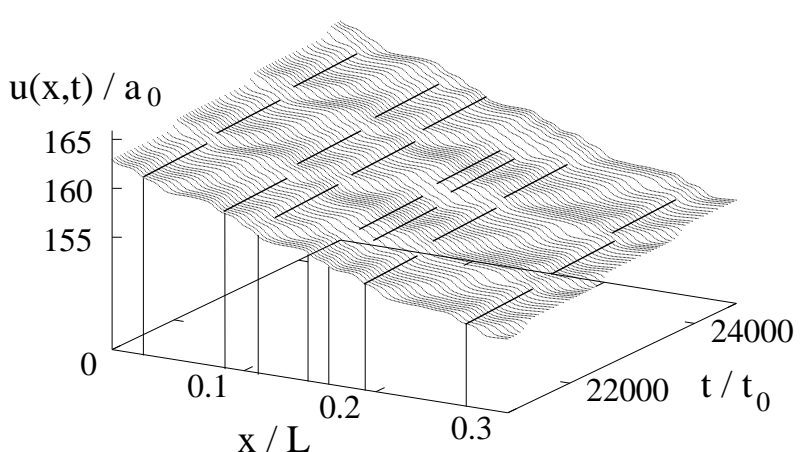

FIG. 10. Time evolution of the displacements $u(x, t)$ in a system of length $L=1000 a_{0}$ for $\chi=-\chi_{c}(0)$ and $f>\tilde{f}_{c}$. Strong pinning sites are indicated by bars parallel to the time axis. Here, interstitials (anti-kinks) travel to the right. At strong pinning sites they collide with pinned kinks. The formerly pinned kinks are released while the previously moving ones become pinned.

and travels until it collides with a defect that is already pinned at a strong pinning site. While the latter becomes released, the former gets pinned. This scenario repeats until a mobile defect has reached the channel exit, see Fig. 10.

In contrast to the force-velocity curve of single systems, which behave as $v \sim\left(f-f_{c}\right)^{\nu}$ with $\nu=1 / 2$, the functional form of the disorder averaged force-velocity curves for finite systems all show upward curvature for $f \gtrsim \tilde{f}_{c}$, which corresponds to $\nu>1$, see Fig. 11. A crossover between exponents similar to the transition $\nu=1 / 2 \rightarrow 1$ occurring in the ordered model at $\chi_{c}(0)$ can not be observed in the disordered case.

Until now we considered the weak disorder limit in agreement with the assumptions made in order to develop the disordered model. If we increase the disorder parameter $W$ beyond the initially assumed limits, we can gain some insight into the depinning properties at large disorder. In Fig. 12 minimum threshold forces $\tilde{f}_{c}$ as a function of disorder strength $W$ for 100 channels with $L=100 a_{0}$, and $\lambda=a_{0}$ are shown for systems without frustration $\chi=0$ and for frustrated systems with $\chi=\chi_{c}(0)$, where we expect the effect of the boundaries to become irrelevant. At $W=0.5$ the disorder is so strong, that a distinction between a commensurate and an incommensurate system cannot be made. We speculate that this indicates a crossover to a depinning transition with true critical behavior as is reported for sandpile models or the Fukuyma-Lee-Rice model for charge density waves.

\section{DISCUSSIONS AND CONCLUSIONS}

In this work we have developed a model for artificial vortex-flow channels motivated by recent experiments.18 20 We have studied the depinning properties of vortices in channels of finite length taking into

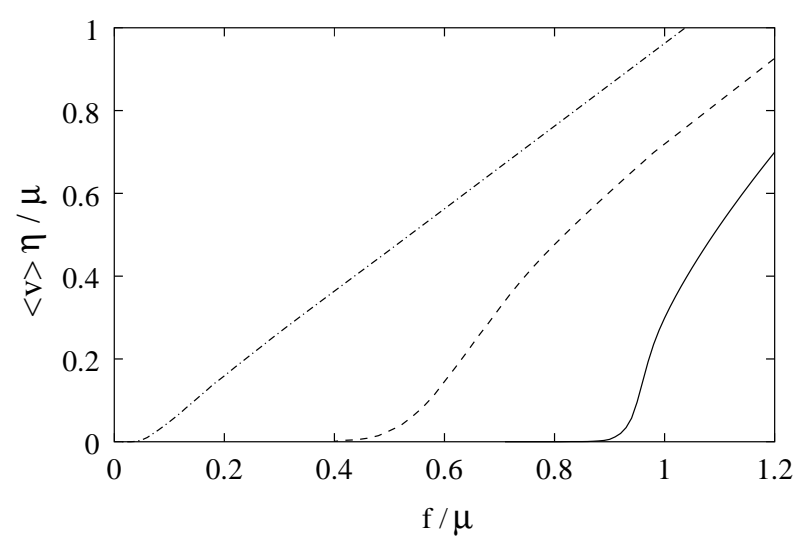

FIG. 11. Disorder averaged $f-v$ curves computed for systems with $L=100 a_{0}$ and $\lambda=a_{0}$. Shown are the $f-v$ characteristics for systems with $\chi=\chi_{c}(0)$ (dash-dotted), $\chi=0.5 \chi_{c}(0)$ (dashed), and $\chi=0$ (solid).

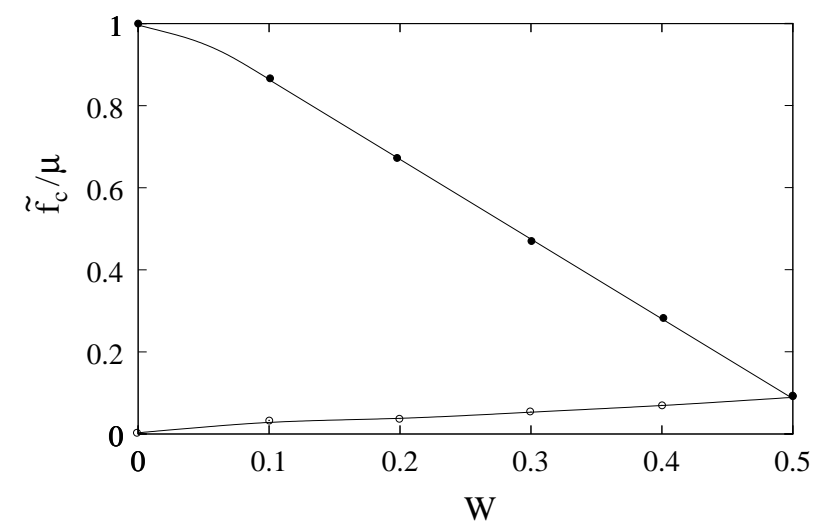

FIG. 12. Numerically obtained minimum threshold force $\tilde{f}_{c}$ as a function of disorder strength $W$ for 100 channels with $L=100 a_{0}$, and $\lambda=a_{0}$. Upper curve: $\chi=0$, lower curve $\chi=\chi_{c}(0)$.

account inhomogeneities such as the sample boundaries and disorder.

Throughout our analysis, we have neglected the influence of thermal or quantum fluctuations on the depinning process. To see that this is well-justified with respect to the experiments that have been performed so far, let us estimate the magnitude of both types of fluctuations in conventional type-II superconductors which have been used to fabricate the channels. The typical pinning energies of topological lattice defects are larger than the vortex self energy, which is about $10 \mathrm{eV}$, whereas the thermal energy is $\sim 10^{-4} \mathrm{eV}$ since the samples are cooled down to $\lesssim 2 K$. Hence, only when the resulting energy barriers are reduced by a factor $\sim 10^{-5}$ one can expect to observe thermal creep of vortex lattice defects. Since the barrier energy scales as $\propto\left(1-j / j_{c}\right)^{3 / 2}$, the width of the current density interval around $j_{c}$ at which thermal creep can be observed is $\Delta j \lesssim 10^{-3} j_{c}$.

To estimate the relevance of quantum creep, we con- 
sider the effective Euclidean action as a function of the current density, $S_{E}^{e f f}(j)$. At equilibrium, $j=0$, $S_{E}^{e f f}(j=0) \approx \xi \hbar^{2} /\left(e^{2} \rho\right)\left(j_{d} / j_{c}\right)^{1 / 2}$, see Ref. Here the coherence length is $\xi \sim 10 \mathrm{~nm}$, the resistivity in the normal state is $\rho \sim 10^{-6} \Omega m$, the depairing current is $j_{d} \sim 10^{9} \mathrm{~A} / \mathrm{m}^{2}$, and the critical current is $j_{c} \sim 10^{6} \mathrm{~A} / \mathrm{m}^{2}$. We find $S_{E}^{e f f}(j=0) / \hbar \sim 10^{3}$. For an overdamped vortex dynamics, which is characteristic for conventional superconductors, $S_{E}^{e f f}(j) / \hbar \sim 10^{3}\left(1-j / j_{c}\right)^{3 / 4}$. Quantum effects become irrelevant for $S_{E}^{e f f}(j) \sim \hbar$, hence $\Delta j \sim 10^{-4} j_{c}$. Thus, in artificial vortex-flow channels made of conventional superconductors thermal and quantum fluctuations only become relevant in an extremely narrow interval around the critical current which has not been resolved in the experiments discussed here. In a first step, we have therefore entirely neglected fluctuations and have studied a purely classical mechanical model at zero temperature.

First, we have considered a perfectly ordered vortex lattice in the channel environment. Starting from a London description of straight vortices in a superconductor, we evaluate the periodic potential experienced by the vortices in the channel. The result, which is strictly valid only at inductions far below $B_{c 2}$ is generalized to higher inductions by taking into account the vortex core and by introducing a field dependent coherence length and penetration depth. We have studied the overdamped dynamics of a chain of interacting vortices in the effective channel potential. In our simplified description we have restricted the considerations to channels with a width of the order of the lattice spacing, $w \sim b_{0}$. We have assumed that the resulting confinement perpendicular to the channel direction is strong such that the transverse motion of the vortices in the channel can be neglected. Further, since in all known channel experiments even for the lowest achievable magnetic inductions $\lambda \gtrsim a_{0}$ holds, to a very good accuracy only the first harmonic of the periodic potential is kept. These assumptions and restrictions allow to determine the coefficients of a driven generalized Frenkel-Kontorova model. For $\lambda \gtrsim a_{0}$, where the typical length $l_{d}$ of a topological defect in the channel is much larger than the lattice spacing $a_{0}$, but still much smaller than the system size $l_{s}, l_{s} \gg l_{d} \gg a_{0}$, the dynamics in the channel is conveniently described by the continuum limit of the Frenkel-Kontorova model known as the driven sine-Gordon model.

After deriving the coefficients of the FrenkelKontorova model, we investigate the CommensurateIncommensurate transition commonly known to occur in this model at thermal equilibrium. Since our focus is on the depinning problem in the absence of fluctuations, we modify the theory. We define a "mechanical" Commensurability-Incommensurability transition. It turns out, that the boundaries of the system play a crucial role if one supposes that the number of static vortices in the strong pinning environment is constant: the purely mechanical Commensurate-Incommensurate tran- sition occurs when the entry barrier for discommensurations at the boundary vanishes.

This concept can be generalized to describe depinning at finite driving forces. The reason is that the entry barrier for discommensurations is reduced when a driving force is applied. The depinning then occurs at frustrations below the zero force threshold frustration, at which the mechanical Commensurate-Incommensurate transition occurs. In this regime, the entry barrier is by far the largest barrier in the system.

Above the zero-force threshold frustration, in the incommensurate regime, the entry barrier has vanished and discommensurations enter the system until the mutual repulsion between the defects prevents new ones to flow in. The extremely small Peierls-Nabarro barrier which may arise due to the discreteness of the system 25 is not taken into account.

The depinning scenario depends on both, on the driving force and on the frustration parameter $\chi=(a-$ $\left.a_{0}\right) / a_{0}$, which measures the mismatch between the lattice constant $a_{0}$ of the channel environment and the preferred lattice spacing in the channel $a$. In the absence of frustration, $\chi=0$, depinning occurs via a trivial homogeneous solution when the barrier of the tilted washboard potential vanishes and the threshold force is given by the amplitude of the sinusoidal channel pinning force, $f_{c}=\mu$. In the presence of frustration, depinning occurs via the formation of topological defects at one of the sample boundaries. For weak frustration, $|\chi| \ll \chi_{c}(0)$, we find $f_{c}=\mu\left\{1-(1 / 2)\left[\sqrt{3} \chi / \chi_{c}(0)\right]^{4 / 3}\right\}$, whereas close to the threshold frustration, $\chi_{c}(0)-|\chi| \ll \chi_{c}(0)$, we obtain $f_{c}=(4 \mu / \pi)\left[1-\chi / \chi_{c}(0)\right]$ and for $|\chi|>\chi_{c}(0)$ the depinning force vanishes, $f_{c}=0$. By performing a numerical integration of the equation of motion we have determined the values of $f_{c}(\chi)$ in between these limits.

Further, we have numerically calculated the forcevelocity curves which correspond to the current-voltage characteristics of the sample. In the commensurate regime, $|\chi|<\chi_{c}(0)$ we find a characteristics typical to a saddle-point bifurcation scenario, $v \sim\left(f-f_{c}\right)^{1 / 2}$, whereas in the incommensurate regime, where the commensurability gap has vanished, the response is linear, $v \sim f$. This behavior is typical for a system with open boundary conditions, where the density of topological defects is a function of both the frustration $\chi$ and the driving force $f$.

In systems with twisted periodic boundary conditions, where the density of topological defects is constant, the force-velocity characteristics is different in the incommensurate regime 20. One observes a linear low-mobility regime for small driving forces, $f \ll \mu$. The slope of the linear part is proportional to the density of topological defects in the system which is fixed by the magnitude of the twist at the ends of the system. The slope of the low-force regime is thus considerably smaller than in the high force regime $f \gg \mu$. For $f \gtrsim \mu$ the curves show a square-root dependence, $v \sim(f-\mu)^{1 / 2}$ and only in the 
high force limit they become linear again. The resulting force-velocity characteristics thus differ significantly from our results in the incommensurate phase, which is linear for all forces.

We conclude that the boundary conditions strongly affect the force-velocity characteristics. The reason is that in frustrated systems the presence of (open) boundaries supports the formation of topological defects which lead to depinning. Further, the entry barriers at the boundaries determine the rate at which defects enter the sample, thus ruling the dynamic behavior entirely. The main problem in determining the behavior of vortex-flow channels is thus to model realistic sample boundaries. Note that other boundary effects than considered here might modify the picture. For example, the vortex lattice of the channel environment may be distorted due to the presence of screening currents along the sample boundary causing a local variation of the frustration. Further, screening currents may lead to Bean-Livingston barriers for vortices which would increase the energy to form a defect at the sample edge. However, the conclusion that transport in artificial vortex-flow channels with a perfectly ordered vortex lattice in the environment is ruled by the entry barriers at the sample boundary persists even if further boundary effects are taken into account.

The picture that depinning occurs only via defect formation at the boundaries does not hold if inhomogeneities are present in the bulk. For example, local distortions of the vortex lattice in the channel environment caused by quenched disorder may generate weak spots that support the formation of vacancy-interstitial pairs at sufficiently large driving force.

To investigate this issue, we have extended the model by accounting for small static displacements $\mathbf{d}(\mathbf{r})$ of the vortices in the channel environment. We assumed the channel environment to be in the elastic limit, $|\nabla \cdot \mathbf{d}| \sim$ $\xi / R_{c} \ll 1$, where $\xi$ is the coherence length measuring the typical displacement within a distance given by the in-plane correlation length $R_{c}$ for lattice distortions. Further, we assumed that close to the channel edges $\lambda \xi / R_{c} \ll a_{0} / 2$ in order to obtain a local approximation for the displacement fields of the static vortices close to the channel edge. Since $\xi / R_{c} \ll 1$ this approximation should be valid as long as the penetration depth is not orders of magnitudes larger than the typical vortex lattice constants. Further, we took only into account longitudinal displacements along the channel edge. For narrow channels, $b_{0} \sim w$, we then obtained a generalized amplitude and phase-disordered Frenkel-Kontorova model which in the continuum limit corresponds to a disordered sine-Gordon model. Transverse displacements imply modifications of the disordered phase and amplitude of the sinusoidal pinning force and to additional pinning force terms. This issue and its consequences for depinning have not been considered here.

In order to gain a basic understanding, we have first investigated depinning caused by a specific longitudinal vortex-lattice distortion field along the channel edges.
Depending on their location, these distortions cause additional local frustration of the system modifying the threshold force for depinning. Distortions at the boundary of the sample affect the entry barrier for topological defects and cause shifts of the $\chi-f$ curve along the $\chi$ axis. Local distortions in the bulk are shown to act as nucleation seeds reducing the threshold force.

Finally, we have studied the effect of small disorderinduced displacements of vortices in the channel environment. We model disorder by uncorrelated relative displacements which are represented by random values that are independent identically distributed according to a box-distribution. In the absence of frustration local lattice distortions in the channel environment lead to an effective channel potential with weak spots. At the weakest spots vacancy-interstitial pairs are formed when reaching the depinning threshold force. In the presence of frustration, a crossover from bulk depinning to boundary depinning occurs when the entry barrier becomes smaller than the smallest bulk pinning barrier. Increasing the frustration the entry barrier is decreased until it becomes smaller than the typical bulk pinning force for kinks due to disorder. Applying a finite driving force that is large enough to overcome the entry barrier, but smaller than the bulk pinning force, topological defects enter the channel and become pinned in the bulk. The finite depinning force in this regime is thus again determined by bulk properties. Increasing the driving force up to the threshold, topological defects which travel some distance until becoming trapped are successively introduced. Above the depinning threshold this leads to a jerky motion with successive depinning and pinning of topological defects.

We obtained the force-velocity characteristics, the threshold force as a function of the frustration, and the threshold force as a function of the disorder for an ensemble of channels with randomly perturbed channel edges. Since the theoretical results are only valid for narrow channels with one mobile row, weak longitudinal disorder, and since the squeezing effects of channel edge currents were not taken into account, the interpretation of the available experimental data remains speculative. It would thus be interesting to extend the model to include these additional properties.

Realizing that the Frenkel-Kontorova model and the sine-Gordon model are equivalent, the question arises, whether the disordered Frenkel-Kontorova model studied here is related to the Fukuyama-Lee-Rice model (FLRM)2829 for charge density waves. This question is especially interesting with respect to the characteristics of the depinning transition. The FLRM and simplified versions have been studied both analytically 3 . 31 and numerically, 3234 mostly in higher dimensions. The FLRM possesses a phase-disordered sinusoidal potential where the phases are chosen randomly from an interval $[-\pi, \pi]$. This model shows critical behavior for $d<4$. Approaching the threshold from below, the critical state is formed by the release of avalanches characterized by typical sizes that diverge with a power-law behavior. 
Above threshold the motion is typically jerky 32 and the velocity shows a power-law behavior $v \sim\left(f-f_{c}\right)^{\nu}$, where $\nu$ depends on the dimensionality of the system.

One is tempted to say that vortex flow channels provide a physical realization of the one-dimensional FLRM. However, we find that the depinning process and the dynamics above threshold strongly depend on the type of boundaries at the sample edges, the strength of the frustration, and the strength and type of disorder. In finite weakly disordered systems as studied in this work three depinning regimes can be identified. Increasing the frustration depinning first takes place via defect nucleation at weak spots in the bulk, then via defect nucleation at the boundary, and finally by releasing pinned pre-existing defects when the frustration is so strong that the entry barrier for defects has become irrelevant.

This is indeed very different compared to the FLRM where system boundaries are not taken into account and the disorder is of a different type. If at all, characteristics of the FLRM like the avalanching below depinning threshold, the roughness of the critical state at threshold, and the jerky motion above the depinning threshold resemble to what we observe in the incommensurate state in the presence of weak disorder.

The main difference to the problem treated here is that the FLRM considers a system close to thermal equilibrium whereas here we are interested in the depinning behavior far from equilibrium. We identify typical configurations that act as sources which produce vacancyinterstitial pairs and thus lower the depinning threshold. These we call weak spots. We find also other configurations that pin the topological defects. The system is static if all topological defects that enter the system via the boundaries or are induced at weak spots are trapped by lattice distortions of the pinning type. Depinning takes place when the density of topological defects becomes larger than the density of pinning sites. It is thus clear that an enhancement of the depinning threshold can only occur in systems with a considerable amount of pinning sites formed by lattice distortions in the channel.

\section{ACKNOWLEDGMENTS}

T. D. acknowledges financial support from the DFGProjekt No. MO815/1-1 and from the Graduierteenkolleg "Physik nanostrukturierter Festkörper", University of Hamburg. R. B. and P. K. are supported by the Nederlandse Stichting voor Fundamenteel Onderzoek der Materie (FOM) and C. M. S. is supported by the Swiss National Foundation under grant 620-62868.00.

\footnotetext{
${ }^{1}$ G. Grüner, Rev. Mod. Phys. 60, 1129 (1988).
}

${ }^{2}$ R. Seshadri and R. M. Westervelt, Phys. Rev. B 46, 5142 (1992); idem, 5150 (1992).

${ }^{3}$ V. M. Vinokur and P. H. Kes and A. E. Koshelev, Physica C 168, 29 (1990).

${ }^{4}$ L. Balents and M. P. A. Fisher, Phys. Rev. Lett. 75, 4270 (1995).

${ }^{5}$ S. Bhattacharya and M. J. Higgins, Physica C 257, 232 (1996).

${ }^{6}$ D. Cule and T. Hwa, Phys. Rev. Lett. 77, 278 (1996).

${ }^{7}$ V. L. Pokrovsky and A. L. Talapov, Phys. Rev. Lett. 42, 65 (1979).

${ }^{8}$ G. Blatter, M. V. Feigel'man, V. B. Geshkenbein, A. I. Larkin and V. Vinokur, Rev. Mod. Phys. 66, 1125 (1994).

${ }^{9}$ S. Bhattacharya and M. J. Higgins, Phys. Rev. Lett. 70, 2617 (1995).

${ }^{10}$ H. J. Jensen, A. Brass, and A. J. Berlinsky, Phys. Rev. Lett. 60, 1676 (1988).

${ }^{11}$ H. J. Jensen, A. Brass, Y. Brechet, and A. J. Berlinsky, Phys. Rev. B 38, 9235 (1988).

${ }^{12}$ H. J. Jensen, Y. Brechet, and A. Brass, J. Low Temp. Phys. 74, 293 (1989).

${ }^{13}$ M. C. Hellerqvist et al., Phys. Rev. Lett. 76, 4022 (1996).

${ }^{14}$ R. Wordenweber, P. H. Kes and C. C. Tsuei, Phys. Rev. B 33, 3172 (1986).

${ }^{15}$ P. Berghuis and P. H. Kes, Phys. Rev. B 47, 262 (1993).

${ }^{16}$ A. C. Marley, M. J. Higgins, and S. Bhattacharya, Phys. Rev. Lett. 74, 3029 (1995).

17 G. DAnna et al., Phys. Rev. Lett. 75, 3521 (1995).

18 A. Pruymboom et al., Phys. Rev. Lett. 60, 1430 (1988).

${ }^{19}$ M. H. Theunissen, E. Van der Drift, and P. H. Kes, Phys. Rev. Lett. 77, 159 (1996).

${ }^{20}$ R. Besseling, R. Niggebrugge, and P. H. Kes, Phys. Rev. Lett. 82, 3144 (1999).

${ }^{21}$ N. Kokubo et al., Phys. Rev. Lett. 88, 247004 (2002); R. Besseling et al., cond-mat/0202485

22 J. Frenkel and T. Kontorova, Zh. Eksp. Teor. Fiz. 8, 1340 (1938); Phys. Z. Sov. 13, 1 (1938).

${ }^{23}$ R. Besseling and P.H. Kes, in preparation; see also http://lions1.leidenuniv.nl/wwwhome/rbesse; R.Besseling, Ph. D. Thesis, Leiden University (2001)

${ }^{24}$ E. H. Brandt, Phys. Rev. B 34, 6514 (1986).

${ }^{25}$ O. M. Braun and Y. S. Kivshar, Phys. Rep. 306, 1 (1998).

${ }^{26}$ S. Aubry, J. Phys. D 7, 240 (1983).

${ }^{27}$ M. Büttiker and R. Landauer, Phys. Rev. A 23, 1397 (1981).

${ }^{28}$ H. Fukuyama, J. Phys. Soc. Jpn. 41, 513 (1976); idem 45, 1474 (1978); H. Fukuyama and P. A. Lee, Phys. Rev. B 17, 535 (1978).

${ }^{29}$ P. A. Lee and T. M. Rice, Phys. Rev. B 19, 3970 (1979).

${ }^{30}$ D. S. Fisher, Phys. Rev. Lett. 50, 1486 (1981); Phys. Rev. B 31, 1396 (1981).

${ }^{31}$ O. Narayan and D. S. Fisher, Phys. Rev. Lett. 68, 3615 (1992); Phys. Rev. B 46, 11520 (1992).

32 S. N. Coppersmith and D. S. Fisher, Phys. Rev. A 38, 6338 (1988).

33 A. A. Middleton and D. S. Fisher, Phys. Rev. Lett. 66, 92 (1991).

${ }^{34}$ C. R. Myers and J. P. Sethna, Phys. Rev. B 47, 11171 (1993). 